\title{
APPROXIMATION BY INVERTIBLE FUNCTIONS OF $H^{\infty}$
}

\author{
ARTUR NICOLAU and DANIEL SUÁREZ
}

\begin{abstract}
We provide an analytic proof that if $H^{\infty}$ is the algebra of bounded analytic functions on the unit disk, $A$ is a Banach algebra and $f: H^{\infty} \rightarrow A$ is a Banach algebras morphism with dense image, then $f\left(\left(H^{\infty}\right)^{-1}\right)$ is dense in $A^{-1}$.
\end{abstract}

\section{Introduction}

If $A$ is a uniform algebra and $n$ is a positive integer, consider the set $U_{n}(A)=$ $\left\{\left(a_{1}, \ldots, a_{n}\right) \in A^{n}: \exists\left(b_{1}, \ldots, b_{n}\right) \in A^{n}\right.$ with $\left.\sum_{j=1}^{n} a_{j} b_{j}=1\right\}$. The Bass stable rank of $A$ is

$$
\begin{aligned}
\operatorname{Bsr} A=\min \{n: \forall a \in & U_{n+1}(A), \exists b \in A^{n} \\
& \text { with } \left.\left(a_{1}+b_{1} a_{n+1}, \ldots, a_{n}+b_{n} a_{n+1}\right) \in U_{n}(A)\right\},
\end{aligned}
$$

with $\operatorname{Bsr} A=\infty$ if there is no such $n$. An alternative and equivalent definition is the minimum integer $n$ such that for every onto morphism of Banach (or uniform) algebras $f: A \rightarrow B$, the induced map from $U_{n}(A)$ into $U_{n}(B)$ is onto (see [4]). This led naturally to define another invariant of uniform algebras, where onto morphisms are replaced by morphisms with dense image [5]. Specifically, the dense stable rank of $A(\operatorname{dsr} A)$ is the minimum $n$ such that for every morphism of uniform algebras $f: A \rightarrow B$ with dense image, the induced application from $U_{n}(A)$ into $U_{n}(B)$ has dense image. In general, Bsr $A \leq \operatorname{dsr} A$.

Let $H^{\infty}$ be the algebra of bounded analytic functions in the unit disk $\mathrm{D}$ with norm $\|f\|=\sup _{z \in \mathrm{D}}|f(z)|$. In [16] Treil proved that Bsr $H^{\infty}=1$ and in [14] the second author proved that dsr $H^{\infty}=1$. The proof of the latter uses part of a very complicated construction in Treil's theorem and topological tools such as Michael's continuous selection theory and Čech cohomology. So, we lacked a purely analytic proof of the equality dsr $H^{\infty}=1$, and it is the purpose of this paper to provide such proof. Our techniques come from giving another twist

Received March 15, 2005; in revised form October 20, 2005. 
to machinery developed over the years by several authors within the theory of $H^{\infty}$.

The maximal ideal space of a uniform algebra $A$ is

$$
M(A)=\{\varphi: A \rightarrow C: \varphi \text { is linear, multiplicative, and } \varphi(1)=1\},
$$

endowed with the weak $*$ topology induced by the dual space of $A$. It is known that $M(A)$ is a compact Hausdorff space, and the Gelfand transform ^: $A \rightarrow C(M(A))$, defined by $\hat{a}(\varphi)=\varphi(a)$, allows us to think of $A$ as a closed subalgebra of $C(M(A))$. For a compact subset $E \subset M(A)$ let $A_{E}$ be the closure in $C(E)$ of the restriction algebra $\left.\hat{A}\right|_{E}$. The $A$-hull of $E$ is

$$
\hat{E}=\left\{\varphi \in M(A):|\hat{a}(\varphi)| \leq \sup _{E}|\hat{a}| \text { for all } a \in A\right\} .
$$

We say that $E$ is $A$-convex if $E=\hat{E}$. It is well-known that $M\left(A_{E}\right)=\hat{E}$ (see [9, p. 39]). As a consequence, we have that $A_{E}=A_{\hat{E}}$ for every closed set $E \subset M(A)$. In [5] there are several equivalent definitions of dsr $A$. It turns out that in the definition of dsr $A$ it is enough to consider only the restriction morphisms from $A$ into $A_{E}$, where $E \subset M(A)$ is a closed $A$-convex set. This means that dsr $A$ is the minimum positive integer such that for every closed $A$-convex set $E \subset M(A)$, the restriction $\left.f \mapsto \hat{f}\right|_{E}$ maps $U_{n}(A)$ into a dense subset of $U_{n}\left(A_{E}\right)$.

Although some of the results in the paper are interesting by themselves, their main purpose for this paper is to give an analytic proof of

Theorem A. dsr $H^{\infty}=1$.

The main point in the proof is the following statement.

Theorem B. Let $0<\eta<\delta<1, \gamma>0$ and $f, g \in H^{\infty}$, with $\|g\| \leq 1$, $\|f\| \leq K$, such that

$$
|f(z)|>\gamma \quad \text { if } \quad|g(z)|<\delta .
$$

Then given $\varepsilon>0$ there exists $F \in H^{\infty}$ such that $|f(z)-F(z)|<\varepsilon$ if $|g(z)|<\eta$ and $C \leq|F(z)|<C^{-1}$, where $C>0$ depends on $K, \varepsilon, \gamma, \delta$ and $\eta$.

The main difficulty in the proof comes from the inner part $I$ of the function $f$. When $I$ is an interpolating Blaschke product, one can use a slight variation of an argument of Treil in [16]. When $I$ is a general inner function, we use a result of approximation by quotients of finite products of interpolating Blaschke products which may be of independent interest (Theorem 3.3). 


\section{Technical lemmas}

First we will collect some well known results which will be used along the paper. Let

$$
\varphi_{z}(w)=\frac{z-w}{1-w \bar{z}}, \quad z, w \in \mathrm{D},
$$

be the automorphism of the disk that interchanges 0 and $z$. The pseudohyperbolic distance between the points $z$ and $w$ in the unit disk is given by $\rho(z, w)=\left|\varphi_{z}(w)\right|$. Schwarz's lemma states that any $f \in H^{\infty}$ with $\|f\| \leq 1$ is a pseudohyperbolic contraction, that is, $\rho(f(z), f(w)) \leq \rho(z, w)$ for any two points $z, w \in \mathrm{D}$. A bounded analytic function in the unit disk is called inner if it has radial limits of modulus 1 along almost every radius. Any inner function can be factorized into a singular inner function, that is an inner function without zeros, and a Blaschke product. Given a sequence $\left\{z_{n}\right\}$ of points in the unit disk D such that

$$
\sum_{n}\left(1-\left|z_{n}\right|\right)<\infty
$$

the Blaschke product with zeros $\left\{z_{n}\right\}$ is defined as

$$
B(z)=z^{m} \prod_{z_{n} \neq 0} \frac{\overline{z_{n}}}{z_{n}} \frac{z_{n}-z}{1-\overline{z_{n}} z} .
$$

Here $m$ is the number of indexes $n$ such that $z_{n}=0$. Let $e^{i \theta}$ be a point in the unit circle and $h>0$. We will denote by $Q=Q\left(e^{i \theta}, h\right)$ the angular square of side-length $\ell(Q)=h$ given by

$$
Q=\left\{r e^{i t}: 0<1-r<h,|t-\theta|<h\right\} .
$$

A positive measure $\mu$ in the unit disk is called a Carleson measure if there exists a constant $C=C(\mu)$ such that $\mu(Q) \leq C \ell(Q)$, for any angular square $Q$ in the unit disk. The infimum of the constants $C$ for which the above estimate holds, is called the Carleson intensity of the measure $\mu$, and will be denoted by $\|\mu\|_{c}$. Carleson measures may be described in conformal invariants terms, as those positive measures $\mu$ for which

$$
\sup _{z \in \mathrm{D}} \int_{\mathrm{D}} \frac{1-|z|^{2}}{|1-\bar{w} z|^{2}} d \mu(w)<\infty .
$$

Moreover, this quantity is equivalent to $\|\mu\|_{c}$. A sequence $\left\{z_{n}\right\}$ of points in the unit disk is called interpolating if for any bounded sequence $\left\{w_{n}\right\}$ of complex numbers, there exists a bounded analytic function $f \in H^{\infty}$ such that $f\left(z_{n}\right)=$ 
$w_{n}$ for all $n$. Let $B$ be the Blaschke product with zeros $\left\{z_{n}\right\}$. These sequences were geometrically described by Carleson as those that satisfy the following two conditions: $\inf _{z_{n} \neq z_{m}} \rho\left(z_{n}, z_{m}\right)>0$, and the measure

$$
\mu_{B}:=\sum_{n}\left(1-\left|z_{n}\right|^{2}\right) \delta_{z_{n}}
$$

is a Carleson measure, where $\delta_{z_{n}}$ is the Dirac measure at $z_{n}$. A Blaschke product is called an interpolating Blaschke product if its zero sequence is an interpolating sequence. Equivalently, the Blaschke product $B$ with zeros $\left\{z_{n}\right\}$ is an interpolating Blaschke product if

$$
\inf _{n}\left(1-\left|z_{n}\right|^{2}\right)\left|B^{\prime}\left(z_{n}\right)\right|>0 .
$$

A Blaschke product $B$ is called a Carleson-Newmann Blaschke product (CNBP for short) if it is a finite product of interpolating Blaschke products, or equivalently, if the measure $\mu_{B}$ is a Carleson measure.

The hyperbolic distance between the points $z, w$ in the unit disk is

$$
h(z, w)=\log \frac{1+\rho(z, w)}{1-\rho(z, w)} .
$$

We use the following notation for the $\varrho$-hyperbolic neighborhood of a set $E$,

$$
\Omega_{\varrho}(E)=\{z \in \mathrm{D}: h(z, E)<\varrho\} .
$$

Also, for a set $E \subset \mathrm{D}$, the radial and the angular projections of $E$ are $\{z /|z|$ : $z \neq 0, z \in E\}$ and $\{|z|: z \in E\}$, respectively. Our first auxiliary result is a variation of a result by Carleson [10, VIII, Thm. 3.2].

Lemma 2.1. Given $0<\delta<1, \varrho>0$ and $\varepsilon>0$, there exists $\eta=$ $\eta(\delta, \varrho, \varepsilon)>0$ with the following property. If $u \in H^{\infty}$, with $\|u\| \leq 1$, and $V \subset \mathrm{D}$ is an angular square such that

$$
\sup \left\{|u(z)|: z \in \Omega_{\varrho}(t(V))\right\} \geq \delta,
$$

where $t(V)$ denotes the top side of $V$, then the length of the radial and angular projections of $\{z \in V:|u(z)|<\eta\}$ is smaller than $\varepsilon \ell(V)$. Moreover, fixed $\varrho>0$ and $\varepsilon>0$, one can take $\eta(\varrho, \delta, \varepsilon)=\delta^{v}$ for some $v=v(\varrho, \varepsilon)>1$.

Proof. Without loss of generality we may assume $\varrho>1$. By hypothesis there are $z_{V} \in t(V)$ and $z_{0}$ such that $h\left(z_{V}, z_{0}\right) \leq \varrho$ and $\left|u\left(z_{0}\right)\right| \geq \delta$. Let $B$ be the hyperbolic disk centered at $z_{V}$ of hyperbolic radius 1 , that is, $B=\{z \in$ 
D : $\left.\rho\left(z, z_{V}\right) \leq r\right\}$, where $r=(e-1) /(e+1)$. We claim that there exists a point $w \in B$ such that

$$
|u(w)| \geq \tilde{\delta},
$$

where

$$
\tilde{\delta}=\tilde{\delta}(\delta, \varrho)=\exp \left(\frac{(\log \delta) \log r^{-1}}{\log \left(e^{2 \varrho}+1\right)-\log \left(e^{2 \varrho}-1\right)}\right) .
$$

To prove (2.1), let us denote by $\omega(z, B, \mathrm{D} \backslash B)$ the harmonic measure from the point $z \in \mathrm{D} \backslash B$ of the set $B$ in the domain $\mathrm{D} \backslash B$. If (2.1) does not hold for any $w \in B$, we would have $\sup _{B}|u|<\tilde{\delta}$ and using the subharmonicity of $\log |u|$, it would follow that

$$
\log |u(z)| \leq(\log \tilde{\delta}) \omega(z, B, \mathrm{D} \backslash B),
$$

for any point $z \in \mathrm{D} \backslash B$. One can easily check that

$$
\omega(z, B, \mathrm{D} \backslash B)=\frac{\log \left|\varphi_{z_{v}}(z)\right|^{-1}}{\log r^{-1}}
$$

and we would have

$$
\log |u(z)| \leq(\log \tilde{\delta}) \frac{\log \left|\varphi_{z_{V}}(z)\right|^{-1}}{\log r^{-1}}
$$

for any $z \in \mathrm{D} \backslash B$. Since $\varrho>1$, one can check that $\delta>\tilde{\delta}$, and using that $\left|u\left(z_{0}\right)\right| \geq \delta$ and $h\left(z_{V}, z_{0}\right) \leq \varrho$, it would follow that

$$
\log \delta \leq(\log \tilde{\delta}) \frac{\log \left|\varphi_{z_{V}}\left(z_{0}\right)\right|^{-1}}{\log r^{-1}} \leq(\log \tilde{\delta}) \frac{\log \frac{e^{e}+1}{e^{e}-1}}{\log r^{-1}} .
$$

But (2.2) says that

$$
\log \delta=(\log \tilde{\delta}) \frac{\log \frac{e^{2 e}+1}{e^{2 e}-1}}{\log r^{-1}},
$$

which contradicts the above inequalities. So (2.1) holds.

The proofs for the angular and radial projections are similar. We write below the proof for the angular projection and at the end we indicate the minor changes necessary to obtain the proof for the radial projection.

Let $\left\{V_{n}\right\}=\left\{V_{k, j}\right\}$ denote the dyadic decomposition of $V$ into angular squares, that is

$$
\begin{aligned}
& V_{k, j} \\
& =\left\{r e^{i \theta}: \frac{2 \pi j}{2^{k}} \ell(V) \leq \theta-\theta_{0}<\frac{2 \pi(j+1)}{2^{k}} \ell(V), 0<1-r \leq 2^{-k} \ell(V)\right\},
\end{aligned}
$$


for $k \geq 0, j=0, \ldots, 2^{k}-1$. Here $\theta_{0}$ is the argument of the center of $V$. If $N$ is a positive integer, consider the symmetrical decomposition of each $T\left(V_{n}\right)=\left\{z \in V_{n}:|z| \leq 1-\ell\left(V_{n}\right) / 2\right\}$, the top half of $V_{n}$, into $N^{2}$ angular rectangles, and denote by $\left\{Q_{j}\right\}$ the family of all the angular rectangles so obtained (for all $n$ ). All these rectangles have comparable pseudohyperbolic diameters, and it is clear that if $d_{N}$ denotes the maximum of these diameters, then $d_{N} \rightarrow 0$ as $N \rightarrow \infty$.

Let $\eta$, with $0<\eta<1$, to be determined later. Among all the rectangles $Q_{j}$ such that $\inf _{Q_{j}}|u|<\eta$, let $\mathscr{F}_{a}$ be a maximal family such that no angular projection of $Q_{j} \in \mathscr{F}_{a}$ is contained in the angular projection of other $Q_{k} \in \mathscr{F}_{a}$. It is clear that the angular projection of $\{z \in V:|u(z)|<\eta\}$ is contained in the angular projection of $\Lambda=\bigcup \mathscr{F}_{a}$. The maximality of $\mathscr{F}_{a}$ implies that the arc-length of $\partial \Lambda$ is a Carleson measure with intensity bounded by an absolute constant. Also, if $Q_{j} \in \mathscr{F}_{a}$ and $z \in Q_{j}$, Schwarz's lemma implies that $|u(z)| \leq \eta+2 d_{N}$. Hence, if $N=N(\eta)$ is chosen big enough so that $d_{N}<\frac{1}{2}\left(\eta^{1 / 2}-\eta\right)$, we get

$$
|u(z)| \leq \eta^{1 / 2} \quad \text { for all } \quad z \in Q_{j}
$$

when $Q_{j} \in \mathscr{F}_{a}$. Suppose that $z \in \partial \Lambda$. Then there is $Q_{k} \in \mathscr{F}_{a}$ with $z \in \partial Q_{k}$. Using that $\log x^{-1} \leq 4(1-x)$ for $1 / 4 \leq x<1$, we see that

$$
\begin{aligned}
\int_{\partial \Lambda} \log \left|\varphi_{z}(\xi)\right|^{-2} & \frac{|d \xi|}{\left(1-|\xi|^{2}\right)} \\
& \leq 8 \int_{\partial \Lambda} \frac{1-|z|^{2}}{|1-\bar{\xi} z|^{2}}|d \xi|+\sum_{\substack{Q_{j} \in \mathscr{F}_{a} \\
\rho\left(Q_{j}, Q_{k}\right) \leq 1 / 4}} \int_{\partial Q_{j}} \log \left|\varphi_{z}(\xi)\right|^{-2} \frac{|d \xi|}{\left(1-|\xi|^{2}\right)} .
\end{aligned}
$$

Since the arc-length of $\partial \Lambda$ is a Carleson measure with intensity bounded by a universal constant, the first summand in the right member is bounded by an absolute constant. Since $d_{N}<1 / 4$, all the 'rectangles' $Q_{j}$ involved in the second summand are contained in the pseudohyperbolic ball of center $z$ and radius $1 / 2$. Also, if for any such $Q_{j}$ we denote by $L_{j}$ its angular projection on the ray $R_{z}=\{\lambda z: 0 \leq \lambda<1\}$, it is easy to check that the integral on $\partial Q_{j}$ is bounded by an absolute constant times the integral on $L_{j}$. Thus, the indexed sum above is bounded by a constant times

$$
\begin{aligned}
\sum_{j} \int_{L_{j}} \log \left|\varphi_{z}(\xi)\right|^{-2} \frac{|d \xi|}{\left(1-|\xi|^{2}\right)} & \leq \int_{\substack{\xi \in R_{z} \\
\rho(\xi, z) \leq 1 / 2}} \log \left|\varphi_{z}(\xi)\right|^{-2} \frac{|d \xi|}{\left(1-|\xi|^{2}\right)} \\
& =\int_{-1 / 2}^{1 / 2} \log |w|^{-2} \frac{d w}{\left(1-|w|^{2}\right)} \leq C
\end{aligned}
$$


where the equality comes from the change of variables $w=\frac{\bar{z}}{|z|} \varphi_{z}(\xi)$ (using the conformal invariance of $\left.|d \xi| /\left(1-|\xi|^{2}\right)\right)$, and $C>0$ is an absolute constant. Putting all together, there is an absolute constant $C_{1} \geq 1$ such that

$$
\int_{\partial \Lambda} \log \left|\varphi_{z}(\xi)\right|^{-2} \frac{|d \xi|}{\left(1-|\xi|^{2}\right)} \leq C_{1} \quad \text { for all } \quad z \in \partial \Lambda .
$$

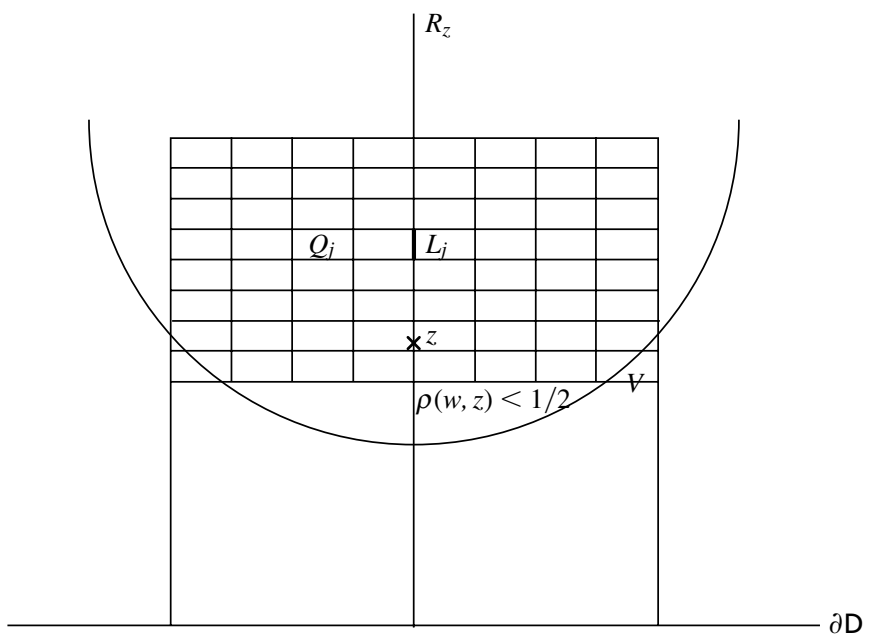

FIGURE 1.

We claim that

$$
\begin{aligned}
C_{1} \log |u(z)| & \leq \log \left(\eta^{1 / 2}\right) \int_{\partial \Lambda} \log \left|\varphi_{z}(\xi)\right|^{-2} \frac{|d \xi|}{\left(1-|\xi|^{2}\right)} \\
& \leq \log \left(\eta^{1 / 2}\right) \int_{\partial \Lambda} \frac{1-|z|^{2}}{|1-\bar{\xi} z|^{2}}|d \xi|
\end{aligned}
$$

for all $z \in \mathrm{D} \backslash \Lambda$. To prove the first inequality observe that since the left member is a subharmonic function and the right member is harmonic on $D \backslash \Lambda$, it is enough to show that it holds for $z \in \partial D \cup \partial \Lambda$. On $\partial \Lambda$ it follows from (2.5) and (2.3) and on $\partial \mathrm{D}$ the left member is $\leq 0$ while the right member vanishes. For the second inequality use that $\log x^{-1} \geq 1-x$ when $0<x<1$.

If $\eta^{1 / 2}<\tilde{\delta}$, (2.3) implies that the point $w$ found in (2.1) is in $\mathrm{D} \backslash \Lambda$. So, putting $z=w$ in (2.6) and using (2.1), we obtain

$$
C_{1} \log \tilde{\delta} \leq \log \left(\eta^{1 / 2}\right) \int_{\partial \Lambda} \frac{1-|w|^{2}}{|1-\bar{\xi} w|^{2}}|d \xi| .
$$


Since $\rho(w, t(V)) \leq(e-1) /(e+1)$, there is an absolute constant $0<C_{2} \leq 1$ such that

$$
\int_{\partial \Lambda} \frac{1-|w|^{2}}{|1-\bar{\xi} w|^{2}}|d \xi| \geq \frac{C_{2}}{\ell(V)} \text { length }(\partial \Lambda),
$$

which together with (2.7) and (2.2) yields

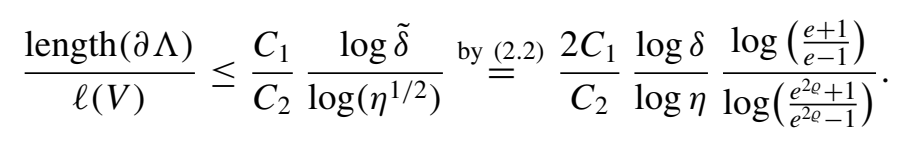

Setting $\eta=\delta^{\nu}$, with

$$
v=v(\varrho, \varepsilon)=\frac{2 C_{1}}{C_{2}} \frac{\log \left(\frac{e+1}{e-1}\right)}{\log \left(\frac{e^{2}+1}{e^{2}-1}\right)} \frac{1}{\varepsilon},
$$

we obtain length $(\partial \Lambda) \leq \varepsilon \ell(V)$, as desired. Observe that since $C_{1} \geq 1$ and $C_{2} \leq 1$, we have $v \geq 2$ for any $0<\varepsilon<1$. Hence, $\eta^{1 / 2}=\delta^{v / 2}<\delta<\tilde{\delta}$, which is the restriction on $\eta$ that we needed to prove (2.7). This proves the lemma for the angular projection.

For the radial projection, instead of $\mathscr{F}_{a}$ we take $\mathscr{F}_{r}$, a maximal family of angular rectangles satisfying $\inf _{Q_{j}}|u|<\eta$, such that no radial projection of $Q_{j} \in \mathscr{F}_{r}$ is contained in the radial projection of other $Q_{k} \in \mathscr{F}_{r} . \operatorname{In}(2.4) L_{j}$ will denote the radial projection of $Q_{j}$ on the circle $C_{z}=\left\{|z| e^{i t}: 0 \leq t<2 \pi\right\}$, and after the change of variables $w=\frac{\bar{z}}{|z|} \varphi_{z}(\xi)$, the last integral in (2.4) will be

$$
\int_{\left|w-\frac{|z|}{1+|z|^{2}}\right|=\frac{|z|}{1+|z|^{2}},|w| \leq 1 / 2} \log |w|^{-2} \frac{|d w|}{\left(1-|w|^{2}\right)},
$$

which is also bounded independently of $z$. The rest of the proof is exactly the same as before. At the end we will obtain the same value of $v$, except for different constants $C_{1}$ and $C_{2}$. The maximum between these two values of $v$ satisfies the lemma.

The next two results on Blaschke products are essentially well known, but simple proofs are presented for the sake of completeness.

Lemma 2.2. Let $B$ be a Blaschke product and $0<\varepsilon<1$. Suppose that $Q \subset \mathrm{D}$ is an angular square such that $|B(w)|>\varepsilon$ for some $w \in \Omega_{\varrho}(T(Q))$, where $T(Q)$ denotes the top half of $Q$. Then there is a constant $C_{Q, \varepsilon}$ depending only on $\varrho$ and $\varepsilon$ such that $\mu_{B}(Q) \leq C_{\varrho, \varepsilon} \ell(Q)$. 
Proof. Let $\left\{z_{n}\right\}$ be the zero sequence of $B$. Using the inequality $\log x^{-1} \geq$ $1-x, 0 \leq x \leq 1$, one deduces that

$$
\log |B(z)|^{-2} \geq \sum_{n} \frac{\left(1-|z|^{2}\right)\left(1-\left|z_{n}\right|^{2}\right)}{\left|1-\bar{z}_{n} z\right|^{2}}
$$

for any $z \in \mathrm{D}$. Hence,

$$
\log \varepsilon^{-2} \geq \sum_{z_{n} \in Q} \frac{\left(1-|w|^{2}\right)\left(1-\left|z_{n}\right|^{2}\right)}{\left|1-\bar{z}_{n} w\right|^{2}} .
$$

Since $h(w, T(Q)) \leq \varrho$, we have $\left|1-\bar{z}_{n} w\right| \leq c(\varrho)\left(1-|w|^{2}\right)$ for any $z_{n} \in Q$. Here $c(\varrho)$ is a constant depending on $\varrho$. So, we deduce

$$
\frac{1-|w|^{2}}{\left|1-\bar{z}_{n} w\right|^{2}} \geq \frac{c_{1}(\varrho)}{\ell(Q)}
$$

where again $c_{1}(\varrho)$ is a constant depending on $\varrho$. Hence

$$
\log \varepsilon^{-2} \geq \frac{c_{1}(\varrho)}{\ell(Q)} \sum_{z_{n} \in Q}\left(1-\left|z_{n}\right|^{2}\right)
$$

and the lemma is proved.

Corollary 2.3. Let $B$ be a Blaschke product and $0<\varepsilon<1$ such that for every zero $z$ of $B$ there exists some point $w$ with $\rho(z, w)<\varrho$ and $|B(w)|>\varepsilon$. Then $B$ is a CNBP such that $\left\|\mu_{B}\right\|_{c} \leq C_{\varrho, \varepsilon}$, where $C_{\varrho, \varepsilon}$ depends only on $\varrho$ and $\varepsilon$.

Proof. Since every square is contained in the union of two dyadic squares of comparable size, it is enough to estimate $\mu_{B}(Q)$ for dyadic squares $Q$. Let $Q$ be a fixed dyadic square and $\mathfrak{F}$ be the family of all the maximal dyadic squares $V \subset Q$ such that there is some zero of $B$ in $T(V)$, the top half of $V$. Clearly, the interiors of these squares are pairwise disjoint, so $\sum_{V \in \mathfrak{S}} \ell(V) \leq \ell(Q)$. Our hypothesis and the previous lemma then say that $\mu_{B}(V) \leq C_{Q, \varepsilon} \ell(V)$, where $C_{\varrho, \varepsilon}$ is as in the lemma. Hence

$$
\mu_{B}(Q)=\mu_{B}\left(\bigcup_{V \in \mathscr{\mathfrak { F }}} V\right) \leq C_{\varrho, \varepsilon} \sum_{V \in \mathfrak{F}} \ell(V) \leq C_{\varrho, \varepsilon} \ell(Q) .
$$

Lemma 2.4. Let $\mu$ be a Carleson measure of intensity at most 1. Given $\varepsilon>0$ there is a constant A depending only on $\varepsilon$ such that

$$
\int_{|z-w| \geq A(1-|z|)} \frac{\left(1-|z|^{2}\right)}{|1-\bar{w} z|^{2}} \mu(w)<\varepsilon
$$


for every $z \in \mathrm{D}$.

Proof. Observe that if $|z-w| \geq 2^{n}\left(1-|z|^{2}\right), n=1,2, \ldots$, one has

$$
\frac{1-|z|^{2}}{|1-\bar{w} z|^{2}} \leq \frac{1}{2^{2 n}\left(1-|z|^{2}\right)}
$$

Fixed $z \in \mathrm{D}$, let

$$
\begin{aligned}
A_{n} & =A_{n}(z) \\
& =\left\{w \in \mathrm{D}: 2^{n+1}\left(1-|z|^{2}\right) \geq|z-w| \geq 2^{n}\left(1-|z|^{2}\right)\right\}, \quad n=1,2, \ldots
\end{aligned}
$$

Then

$$
\sum_{n \geq k} \int_{A_{n}} \frac{1-|z|^{2}}{|1-\bar{w} z|^{2}} d \mu(w) \leq \sum_{n \geq k} \frac{\mu\left(A_{n}\right)}{2^{2 n}\left(1-|z|^{2}\right)} .
$$

Since $\mu\left(A_{n}\right) \leq C 2^{n+1}\left(1-|z|^{2}\right)$ for an absolute constant $C>0$, the last sum can be bounded by $C \sum_{n \geq k} 1 / 2^{n-1}$, and the result follows with $A=2^{k}>$ $4 C / \varepsilon$.

Let $d A$ denote the normalized area measure on $\mathrm{D}, \partial$ and $\bar{\partial}$ the CauchyRiemann operators, $\nabla$ be the gradient operator and $\Delta=\partial \bar{\partial}$ (i.e.: a quarter of the standard Laplacian operator). The next result was essentially given by Treil [16].

LEMMA 2.5. Let $\Psi \in C^{\infty}$ (D) such that for some positive constants $K_{1}, K_{2}$, $K_{3}$ one has

(1) $\||\Psi(z)| d A(z)\|_{c} \leq K_{1}$,

(2) $\|(1-|z|)|\partial \Psi(z)| d A(z)\|_{c} \leq K_{2}$,

(3) $\sup _{z \in \mathrm{D}}(1-|z|)^{2}|\partial \Psi(z)| \leq K_{3}$ and $\sup _{z \in \mathrm{D}}(1-|z|)|\Psi(z)| \leq K_{3}$.

Then there exists $a \in C^{2}(\mathrm{D})$ such that $\bar{\partial} a=\Psi$ and $\sup _{z \in \mathrm{D}}|a(z)| \leq$ $C\left(K_{1}, K_{2}, K_{3}\right)$.

Proof. For any positive integer $n$ let $\phi_{n}: \mathrm{D} \rightarrow[0,1]$ be a $C^{\infty}$ function such that

$$
\phi_{n}(z)= \begin{cases}1 & \text { if }|z| \leq 1-2^{-n} \\ 0 & \text { if }|z|>1-2^{-(n+1)}\end{cases}
$$

and $\left|\nabla \phi_{n}(z)\right| \leq C 2^{n}$ for some absolute constant $C>0$. So, $(1-|z|)\left|\nabla \phi_{n}(z)\right|$ $\leq C$. Condition (1) implies that there exists a function $b_{n} \in C^{2}(\overline{\mathrm{D}})$ with $\bar{\partial} b_{n}=\phi_{n} \Psi$ and $\left\|b_{n}\right\|_{L^{\infty}(\partial \mathrm{D})}<C\left(K_{1}\right)$ (see [9, p. 230]). Hence, the Poisson 
integral $u_{n}$ of $b_{n}$ is also bounded by $C\left(K_{1}\right)$ in the whole closed unit disk. We have $b_{n}(z)=u_{n}(z)-g_{n}(z)$, where

$$
g_{n}(z)=\int_{\mathrm{D}} \Delta b_{n}(w) \log \left|\frac{1-\bar{z} w}{z-w}\right|^{2} d A(w)
$$

Since $\Delta b_{n}=\partial\left(\phi_{n} \Psi\right)$, we have

$$
\left|g_{n}(z)\right| \leq \int_{\mathrm{D}}\left[\frac{C}{1-|w|}|\Psi(w)|+|\partial \Psi(w)|\right] \log \left|\frac{1-\bar{z} w}{z-w}\right|^{2} d A(w) .
$$

Split the above integral into $\int_{D(z)}+\int_{D \backslash D(z)}$, where $D(z)=\{w: \rho(w, z) \leq$ $1 / 2\}$. By (3)

$$
\begin{aligned}
\int_{D(z)}\left[\frac{C|\Psi(w)|}{1-|w|}+|\partial \Psi(w)|\right] \log & \left|\frac{1-\bar{z} w}{z-w}\right|^{2} d A(w) \\
& \leq \int_{D(z)} \frac{\left(C K_{3}+K_{3}\right)}{(1-|w|)^{2}} \log \left|\frac{1-\bar{z} w}{z-w}\right|^{2} d A(w) .
\end{aligned}
$$

The conformal invariance of the measure $\left(1-|w|^{2}\right)^{-2} d A(w)$ immediately shows that the last integral is uniformly bounded by some absolute constant times $K_{3}$. Using the elementary estimate $\log x^{-2}<c\left(1-x^{2}\right)$ if $1 / 2<x<1$, the second integral can be estimated by

$$
\int_{D \backslash D(z)}\left[\frac{C|\Psi(w)|}{1-|w|}+|\partial \Psi(w)|\right] c \frac{\left(1-|z|^{2}\right)\left(1-|w|^{2}\right)}{|1-\bar{z} w|^{2}} d A(w),
$$

which by (1) and (2) is bounded by an absolute constant times $K_{1}+K_{2}$. Hence, we deduce that

$$
\sup _{z \in \mathrm{D}}\left|b_{n}(z)\right| \leq C\left(K_{1}, K_{2}, K_{3}\right)
$$

Now we may consider a weak star limit $b \in L^{\infty}$ (D) of a suitable subsequence of $b_{n}$. Then $\|b\|_{L^{\infty}(\mathrm{D})} \leq C\left(K_{1}, K_{2}, K_{3}\right)$ and $\bar{\partial} b=\Psi$ in the sense of distributions. Since $\Psi \in C^{\infty}(\mathrm{D})$, applying the hypoellipticity of the operator $\bar{\partial}$ (see [7, p. 270]), we obtain that in fact $b \in C^{\infty}(\mathrm{D})$.

\section{Two approximation results}

A more general, but non-quantitative version of the next theorem can be found in [15]. Given a set $E$ in the unit disk and a function $f$ defined on $E$, we denote $\|f\|_{E}=\sup \{|f(z)|: z \in E\}$. 
Theorem 3.1. Let $g \in H^{\infty}$ with $\|g\|=1$ and $0<\alpha<\beta<1$. Given an analytic function $f$ on the set $\{|g|<\beta\}$ with $\|f\|_{\{|g|<\beta\}} \leq 1$ and $\varepsilon>0$, there exists $F \in H^{\infty}$ such that

$$
\|F-f\|_{\{|g|<\alpha\}}<\varepsilon
$$

with $\|F\| \leq C$, where $C=C(\alpha, \beta, \varepsilon)$.

Proof. For $k=1,2,3,4$ put $\gamma_{k}=\alpha+k(\beta-\alpha) / 5$. So,

$$
\alpha<\gamma_{1}<\gamma_{2}<\gamma_{3}<\gamma_{4}<\beta .
$$

By Bishop's construction in [1] there is an open region $R \subset \mathrm{D}$ such that

(1) $\left\{|g|<\gamma_{2}\right\} \subset R \subset\left\{|g|<\gamma_{3}\right\}$,

(2) $\partial R$ is a Carleson contour whose associated arc-length Carleson measure $\lambda_{\partial R}$ satisfies $\left\|\lambda_{\partial R}\right\|_{c} \leq C$, where $C=C\left(\gamma_{2}, \gamma_{3}\right)$.

Since $\partial R \subset\left\{\gamma_{2} \leq|g| \leq \gamma_{3}\right\}$, Schwarz's Lemma implies that there is a positive constant $\eta$ depending on $\gamma_{j}$ for $j=1, \ldots, 4$, such that

$$
\rho\left(\partial R,\left\{|g| \leq \gamma_{1}\right\} \cup\left\{|g| \geq \gamma_{4}\right\}\right) \geq \eta .
$$

Hence, the characteristic function of $R$ can be modified in a $\eta / 2$-pseudohyperbolic neighborhood of $\partial R$ in order to obtain a $C^{\infty}$ function $0 \leq \Phi \leq 1$ such that

(a) $\Phi \equiv 1$ on $\left\{|g| \leq \gamma_{1}\right\}$,

(b) $\Phi \equiv 0$ on $\left\{|g| \geq \gamma_{4}\right\}$,

(c) $(1-|z|)^{2}|\Delta \Phi(z)| \leq C / \eta^{2}$ and $(1-|z|)|\bar{\partial} \Phi(z)| \leq C / \eta$, where $C>0$ is an absolute constant,

(d) $|\bar{\partial} \Phi(z)| d A(z)$ and $(1-|z|)|\Delta \Phi(z)| d A(z)$ are Carleson measures with Carleson norms bounded by a constant depending only on $\eta$ and $\left\|\lambda_{\partial R}\right\|_{c}$.

For every positive integer $n$, consider the $\bar{\partial}$-equation

$$
\bar{\partial} a_{n}=\frac{1}{n}\left(\gamma_{1} / g\right)^{n} f \bar{\partial} \Phi .
$$

Let $\Psi_{n}$ denote the right member of (3.3). We shall see that $\Psi_{n}$ fits into Lemma 2.5 with constants $K_{j}(j=1,2,3)$ that depend only on $\alpha$ and $\beta$ (independently of $n$ ). Since the support of $\bar{\partial} \Phi$ is contained in $\left\{\gamma_{1} \leq|g| \leq \gamma_{4}\right\}$ then 
$\left|\left(\gamma_{1} / g\right)^{n} f\right| \leq 1$ on supp $\bar{\partial} \Phi$. Hence, since $|\bar{\partial} \Phi(w)| d A(w)$ is a Carleson measure, we deduce condition (1) in Lemma 2.5. Moreover, (3.2) implies that

$$
\rho\left(\operatorname{supp} \bar{\partial} \Phi,\left\{|g| \leq \gamma_{1}\right\} \cup\left\{|g| \geq \gamma_{4}\right\}\right) \geq \eta / 2 .
$$

Thus, there is a positive constant $c_{\eta}$ depending only on $\eta$ such that whenever $z \in \operatorname{supp} \bar{\partial} \Phi$, the closed (Euclidean) disk of center $z$ and radius $c_{\eta}(1-|z|)$ is contained in $\left\{\gamma_{1} \leq|g| \leq \gamma_{4}\right\}$. The Cauchy integral formula for $f^{\prime}(z)$ over the boundary of this ball easily yields

$$
\left|f^{\prime}(z)\right| \leq \frac{1}{c_{\eta}(1-|z|)} \quad \text { on } \quad \operatorname{supp} \bar{\partial} \Phi .
$$

We have

$$
\begin{aligned}
\left|\partial \Psi_{n}\right| & =\left|\left[\frac{1}{n}\left(\frac{\gamma_{1}}{g}\right)^{n} f^{\prime}-\left(\frac{\gamma_{1}}{g}\right)^{n}\left(\frac{g^{\prime}}{g}\right) f\right] \bar{\partial} \Phi+\frac{1}{n}\left(\frac{\gamma_{1}}{g}\right)^{n} f \partial \bar{\partial} \Phi\right| \\
& \leq\left[\frac{1}{n}\left|f^{\prime}\right|+\frac{\left|g^{\prime}\right|}{\gamma_{1}}\right]|\bar{\partial} \Phi|+\frac{1}{n}|\partial \bar{\partial} \Phi|,
\end{aligned}
$$

where the inequality holds because $|g| \geq \gamma_{1}$ on supp $\bar{\partial} \Phi$. Since $(1-|z|)\left|g^{\prime}(z)\right|$ $\leq\|g\|=1$, then properties (c) and (d) of $\Phi$ imply that $\Psi_{n}$ satisfies hypotheses (2) and (3) of Lemma 2.5 with constants $K_{1}, K_{2}$ and $K_{3}$ depending only on $\gamma_{1}$, $\eta$ and $\left\|\lambda_{\partial R}\right\|_{c}$. These three quantities depend on the $\gamma_{j}$ 's, which in turn only depend on $\alpha$ and $\beta$, as claimed. Thus, the lemma says that the equation (3.3) has a solution $a_{n}$ with $\sup _{\mathrm{D}}\left|a_{n}\right| \leq C(\alpha, \beta)$. Hence $F_{n}:=f \Phi-n\left(g / \gamma_{1}\right)^{n} a_{n}$ is analytic on $\mathrm{D},\left\|F_{n}\right\| \leq 1+n \gamma_{1}^{-n} C(\alpha, \beta)$, and on the set $\{|g|<\alpha\}$ :

$$
\begin{aligned}
\left|F_{n}-f\right| & =\left|f \Phi-n\left(g / \gamma_{1}\right)^{n} a_{n}-f\right| \\
& =n\left|g / \gamma_{1}\right|^{n}\left|a_{n}\right| \\
& \leq n\left(\alpha / \gamma_{1}\right)^{n} C(\alpha, \beta)<\varepsilon
\end{aligned}
$$

if we fix some $n=n(\alpha, \beta, \varepsilon)$ big enough.

The proof of the next lemma is a modification of the construction in [11]. A feature to remark is that the constant $M$ in the lemma does not depend on any of the other parameters.

Lemma 3.2. There exists a positive integer $M$ with the following property: for any Blaschke product $u$ and numbers $0<\beta<1$ and $\varepsilon>0$, there exists a factorization $u=u_{0} u_{1} \ldots u_{M}$ and Carleson-Newman Blaschke products $B_{p}$ and $B_{p}^{*}$ for $1 \leq p \leq M$, such that 
(a) $\left|u_{p}(z)-\frac{B_{p}^{*}(z)}{B_{p}(z)}\right|<\varepsilon$ on $\{|u(z)|>\beta\}$,

(b) $\left\|\mu_{u_{0}}\right\|_{c},\left\|\mu_{B_{p}}\right\|_{c},\left\|\mu_{B_{p}^{*}}\right\|_{c} \leq c_{1}(\varepsilon, \beta)$,

(c) $\left|B_{p}(z)\right|,\left|B_{p}^{*}(z)\right| \geq c_{2}(\varepsilon, \beta)>0$ on $\{|u(z)|>\beta\}$,

where $c_{1}(\varepsilon, \beta)$ and $c_{2}(\varepsilon, \beta)$ only depend on $\varepsilon$ and $\beta$.

Proof. By a Möbius transformation of the variable $z$, we can assume without loss of generality that $|u(0)| \geq \beta$. Let $M>0$ be a positive integer to be determined later. For $n \geq 1$ an integer, consider the angular squares

$$
Q_{n, j}=\left\{r e^{i \theta}: \frac{2 \pi j}{M^{n}} \leq \theta \leq \frac{2 \pi(j+1)}{M^{n}}, 1-\frac{1}{M^{n}} \leq r<1\right\},
$$

where $j=0, \ldots, M^{n}-1$. Put

$$
T\left(Q_{n, j}\right)=\left\{z \in Q_{n, j}:|z| \leq 1-\ell\left(Q_{n, j}\right) / M\right\},
$$

where $\ell\left(Q_{n, j}\right)=1 / M^{n}$. Notice that $T\left(Q_{n, j}\right)$ is the closure of

$$
Q_{n, j} \backslash \bigcup_{k}\left\{Q_{n+1, k}: Q_{n+1, k} \subset Q_{n, j}\right\} .
$$

The hyperbolic diameters $\operatorname{diam}_{h}\left(T\left(Q_{n, j}\right)\right)$ are roughly the same for all $n, j$. Let $\alpha$, with $0 \leq \alpha \leq \beta$, to be fixed later. Next we will run a stopping time argument to construct a Carleson contour.

Step 1. Take $\varrho=\varrho(M)>\operatorname{diam}_{h}\left(T\left(Q_{n, j}\right)\right)$ for all $n$ and $j$, to be determined later. Suppose that $Q_{n, j}$ is maximal with respect to the property

$$
\sup _{\Omega_{2 \ell}\left(T\left(Q_{n, j}\right)\right)}|u(z)|<\alpha,
$$

and then rename the $M$ squares $Q_{n+1, k} \subset Q_{n, j}$ by $S_{n, j}^{p}, p=1, \ldots, M$. Our condition on $\varrho$ implies that

$$
\sup \left\{|u(z)|: z \in \Omega_{\varrho}\left(T\left(S_{n, j}^{p}\right)\right)\right\}<\alpha .
$$

Step 2. Denote by $V_{k}$ the maximal squares $Q_{m, l} \subset S_{n, j}^{p}$ such that

$$
\sup _{\Omega_{\varrho}\left(T\left(Q_{m, l}\right)\right)}|u(z)| \geq \beta .
$$

Since $\left|u\left(e^{i \theta}\right)\right|=1$ a.e., then

$$
\sum \ell\left(V_{k}\right)=\ell\left(S_{n, j}^{p}\right)
$$


where the sum is taken over all $V_{k}$ contained in $S_{n, j}^{p}$. Put $R_{n, j}^{p}=S_{n, j}^{p} \backslash \bigcup\left\{V_{k}\right.$ : $V_{k} \subset S_{n, j}^{p}$. Now for each $V_{k}$ consider the maximal squares $Q_{m, l} \subset V_{k}$, such that

$$
\sup _{\Omega_{2 Q}\left(T\left(Q_{m, l}\right)\right)}|u(z)|<\alpha .
$$

Hence, Lemma 2.1 tells us that if $\alpha=\alpha(\beta, \varrho)$ is small enough (in particular, much smaller than $\beta$ ), then

$$
\sum \ell\left(Q_{m, l}\right) \leq \frac{1}{2} \ell\left(V_{k}\right)
$$

where the sum is taken over all $Q_{m, l} \subset V_{k}$ such that $Q_{m, l}$ satisfies (3.8). Condition (3.8) is condition (3.4), so we repeat Step 1 with $Q_{m, l}$ and so forth. So, by induction, we obtain nested families of $M$-adic angular squares which we denote as $S_{n, j}^{p}$ if they appear in Step 1 and $V_{k}$ if they do in Step 2. Also, we consider the intermediate region $R_{n, j}^{p}=S_{n, j}^{p} \backslash \bigcup\left\{V_{k}: V_{k} \subset S_{n, j}^{p}\right\}$. For $p=1, \ldots, M$ take $\Lambda_{p}=\bigcup_{n, j} R_{n, j}^{p}$. By (3.6),

$$
\Omega_{\varrho}\left(\bigcup_{1 \leq p \leq M} \Lambda_{p}\right) \subset\{|u(z)|<\beta\} .
$$

If $z \notin \bigcup_{1 \leq p \leq M} \Lambda_{p}$ there are two possibilities: either

$$
z \in V_{k} \backslash \bigcup\left\{Q \subset V_{k}: Q \text { satisfies (3.4) }\right\}
$$

for some $V_{k}$ or

$$
z \in T\left(Q_{n, j}\right)
$$

where $Q_{n, j}$ is one of the maximal squares satisfying (3.4). In the first case there is some $w=w(z)$ such that $h(w, z) \leq \operatorname{diam}_{h}(T(Q))+2 \varrho$ and $|u(w)| \geq \alpha$. In the second case, we observe that, by the maximality of $Q_{n, j}$, the father of $Q_{n, j}$ does not satisfy (3.4) and hence there exists a point $w=w(z)$ such that $h(w, z) \leq 2 \operatorname{diam}_{h}(T(Q))+2 \varrho$ and $|u(w)| \geq \alpha$. So, in both cases there exists $w$, with $h(w, z) \leq 4 \varrho$, such that $|u(w)| \geq \alpha$. Let $u_{0}$ be the Blaschke product with zeros

$$
\left\{z: u(z)=0 \text { and } z \notin \bigcup_{1 \leq p \leq M} \Lambda_{p}\right\} .
$$

By the above paragraph and Corollary 2.3, $u_{0}$ is a CNBP with $\left\|\mu_{u_{0}}\right\|_{c} \leq C_{Q, \alpha}$.

Define $u_{p}$, for $1 \leq p \leq M$, as the Blaschke product whose zeros are the zeros of $u$ that belong to the closure of $\Lambda_{p}$. Observe that by (3.7) the linear 
Lebesgue measure $\left|\partial \Lambda_{p} \cap \partial \mathrm{D}\right|=0$, and by (3.7) and (3.9) the arc-length of $\partial \Lambda_{p}$ is a Carleson measure with intensity bounded by an absolute constant $K_{0}$ (independent of $\varrho, \alpha, \beta$ or $M$ ).

Now, fixed $\delta \geq 0$, we pick points $z_{v}$ in $\partial \Lambda_{p}$ located each $\delta / 4$ pseudohyperbolic units. More concretely, fixed $p=1 \ldots M$, pick points $z_{\nu}$ of $\partial \Lambda_{p}$ so that for every $z \in \partial \Lambda_{p}$ there is $z_{v}$ such that $\rho\left(z_{v}, z\right)<\delta / 2$, and $\rho\left(z_{n}, z_{k}\right) \geq \delta / 4$ if $n \neq k$. Let $B_{p}$ be the Blaschke product with zeros $\left\{z_{v}\right\}$. Then $\left\|\mu_{B_{p}}\right\|_{c}$ is bounded by some constant of the form $C K_{0} / \delta$, where $C$ is an absolute constant (and $K_{0}$ is as above, also an absolute constant). We write $K_{1}=C K_{0}$.

We next show that there is an absolute constant $C_{2}$ such that

$$
\sup \left\{\left|B_{p}(z)\right|: z \in \Lambda_{p}\right\}<e^{-C_{2} / \delta} .
$$

Indeed, if $Q(z)$ is the angular square centered at $z$ (for completeness, take $Q(z)=\mathrm{D}$ if $|z| \leq 1 / 2)$, using the inequality $\log (1 / x)>1-x$ for $0<x<1$, we have

$$
\log \frac{1}{\left|B_{p}(z)\right|^{2}}>\sum_{\nu} \frac{\left(1-|z|^{2}\right)\left(1-\left|z_{\nu}\right|^{2}\right)}{\left|1-\overline{z_{\nu}} z\right|^{2}} \geq \frac{C}{(1-|z|)} \sum_{z_{\nu} \in Q(z)}\left(1-\left|z_{\nu}\right|^{2}\right),
$$

for some absolute constant $C>0$. Since $1-\left|z_{v}\right| \geq c\left|z_{v+1}-z_{v}\right| / \delta$ for another absolute constant $c>0$, the last sum is bigger than a fixed proportion of the length of $Q(z) \cap \partial \Lambda_{p}$ divided by $\delta$, and since $z \in \Lambda_{p}$, this length is bigger than a fixed fraction of $1-|z|$. This proves (3.11). Since the Carleson norm of the measure $\left(\delta / K_{1}\right) \mu_{B_{p}}$ is at most 1 , applying Lemma 2.4 we see that there is an absolute constant $A$ big enough so that

$$
\int_{\{w:|z-w| \geq A(1-|z|)\}} \frac{\left(1-|z|^{2}\right)}{|1-\bar{w} z|^{2}} d \mu_{B_{p}}(w)<\frac{C_{2}}{20 \delta}
$$

for every $z \in \mathrm{D}$. Also, we can assume that $A>2^{6}$. In order to simplify notation, for $z, \omega \in \mathrm{D}$ we write

$$
a(z, \omega)=1-\rho(z, \omega)^{2}=\frac{\left(1-|z|^{2}\right)\left(1-|\omega|^{2}\right)}{|1-\bar{\omega} z|^{2}} .
$$

Let $\left\{w_{\nu}\right\}$ be the zero set of $B_{p} u_{p}$. Then

$$
\log \frac{1}{\left|\left(B_{p} u_{p}\right)(z)\right|^{2}} \leq\left(1+2 \log \frac{1}{\lambda}\right) \sum_{\nu} a\left(z, w_{\nu}\right)
$$

if $\rho\left(z, w_{v}\right) \geq \lambda>0$ for all $v[10$, VII, Lemma 1.2], and

$$
\left(1-|z|^{2}\right) \frac{\left(B_{p} u_{p}\right)^{\prime}}{B_{p} u_{p}}(z)=\sum_{\nu} a\left(z, w_{\nu}\right)\left(\frac{1-w_{\nu} \bar{z}}{z-w_{\nu}}\right)
$$




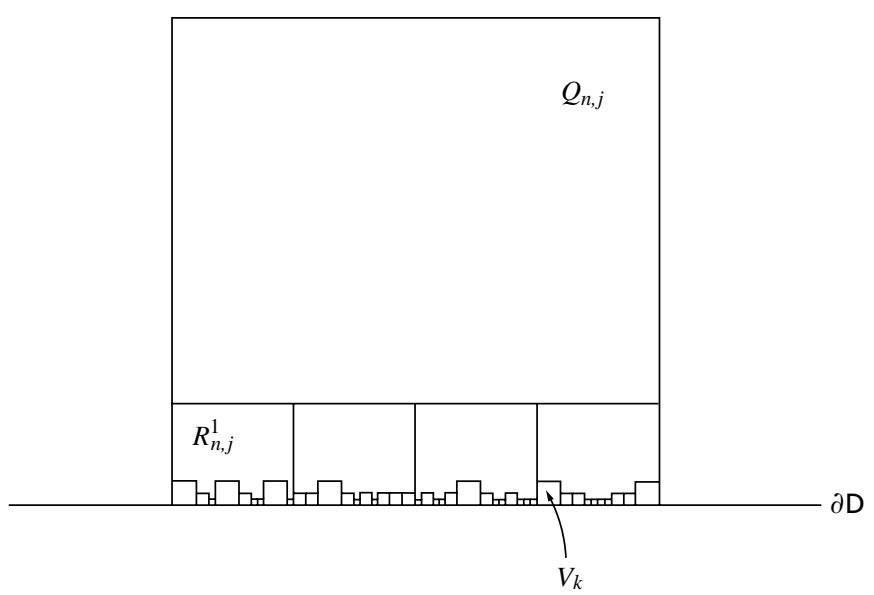

FIGURE 2.

if $z \notin\left\{w_{v}\right\}$. Take

$$
\lambda=\lambda(\varrho)=\frac{e^{\varrho}-1}{e^{\varrho}+1} .
$$

Let $z \in \mathrm{D}$ be such that

$$
\left.\inf _{\xi \in \Lambda_{p}} h(z, \xi) \geq \varrho \quad \text { (i.e.: } \inf _{\xi \in \Lambda_{p}} \rho(z, \xi) \geq \lambda\right)
$$

If $\varrho=\varrho(M)$ is big enough, we have that whenever $z_{v} \in R_{n, j}^{p}$ satisfies $\left|z-z_{v}\right|<$ $A(1-|z|)$, then $\ell\left(S_{n, j}^{p}\right)<\frac{1}{M}(1-|z|)$. Put $\Delta(z)=\{\omega \in \mathrm{D}:|z-\omega|<$ $A(1-|z|)\}$ and let $I_{z}$ be the radial projection of $\Delta(z)$ into $\partial \mathrm{D}$. It is clear that there is some absolute constant $C_{0}>1$ such that $\left|I_{z}\right| \leq C_{0}(1-|z|)$.

For $p$ fixed, consider the family $\mathcal{M}_{z}$ of all the maximal angular squares $S_{n, j}^{p}$ such that there is some $z_{v} \in R_{n, j}^{p} \cap \Delta(z)$. Then the above paragraph yields

$$
\sum_{S_{n, j}^{p} \in \mathscr{M}_{z}} \ell\left(S_{n, j}^{p}\right) \leq \frac{(1-|z|)}{M}+\left|I_{z}\right| \sum_{k=1}^{\infty} M^{-k} \leq \frac{C_{0}^{\prime}}{M}(1-|z|) .
$$

Hence,

$$
\begin{aligned}
\sum_{w_{v} \in \Delta(z)} a\left(z, w_{\nu}\right) \leq \sum_{w_{\nu} \in \Delta(z)} \frac{2\left(1-\left|w_{\nu}\right|^{2}\right)}{1-|z|} & =\frac{2}{1-|z|} \sum_{w_{\nu} \in S_{n, j}^{p} \in \mathscr{M}_{z}}\left(1-\left|w_{\nu}\right|^{2}\right) \\
& =\frac{2}{1-|z|}\left(J_{1}+J_{2}\right),
\end{aligned}
$$


where

$$
J_{1}=\sum_{S_{n, j}^{p} \in M_{z}} \mu_{B_{p}}\left(S_{n, j}^{p}\right) \quad \text { and } \quad J_{2}=\sum_{S_{n, j}^{p} \in \mathscr{M}_{z}} \mu_{u_{p}}\left(S_{n, j}^{p}\right) .
$$

Since $\left\|\mu_{B_{p}}\right\|_{c} \leq K_{1} / \delta$, equation (3.16) gives

$$
J_{1} \leq \frac{K_{1}}{\delta} \sum_{S_{n, j}^{p} \in \mathscr{M}_{z}} \ell\left(S_{n, j}^{p}\right) \leq \frac{K_{1}}{\delta} \frac{C_{0}^{\prime}}{M}(1-|z|) .
$$

Since by construction the grandfather square of $S_{n, j}^{p}$ is at $h$-distance at most $2 \varrho$ of a point $w$ with $|u(w)| \geq \alpha$, Lemma 2.2 tells us that $\mu_{u_{p}}\left(S_{n, j}^{p}\right) \leq$ $C(\varrho, \alpha) \ell\left(S_{n, j}^{p}\right)$. So (3.16) gives

$$
J_{2} \leq C(\varrho, \alpha) \sum_{S_{n, j}^{p} \in \mathscr{M}_{z}} \ell\left(S_{n, j}^{p}\right) \leq C(\varrho, \alpha) \frac{C_{0}^{\prime}}{M}(1-|z|) .
$$

Consequently

$$
\sum_{w_{v} \in \Delta(z)} a\left(z, w_{\nu}\right) \leq \frac{C_{1}}{\delta M}+C^{\prime}(\varrho, \alpha, M),
$$

where $C_{1}$ is an absolute constant and $C^{\prime}(\varrho, \alpha, M)$ only depends on $\varrho, \alpha$ and $M$. On the other hand, if $w_{v} \notin \Delta(z)$, that is $\left|z-w_{v}\right| \geq A(1-|z|)$, then

$$
|z| \geq 1-\frac{\left|z-w_{\nu}\right|}{A} \geq 1-\frac{2}{A} .
$$

Since $A \geq 2^{6}$ we obtain

$$
\begin{aligned}
\left|\sum_{w_{\nu} \notin \Delta(z)} a\left(z, w_{\nu}\right)\left(\frac{1-w_{\nu} \bar{z}}{z-w_{\nu}}\right)\right| & =\left|\sum_{w_{\nu} \notin \Delta(z)} a\left(z, w_{\nu}\right)\left[\bar{z}+\frac{1-|z|^{2}}{z-w_{\nu}}\right]\right| \\
& \geq \sum_{w_{\nu} \notin \Delta(z)} a\left(z, w_{\nu}\right)\left[|z|-\frac{1-|z|^{2}}{\left|z-w_{\nu}\right|}\right] \\
& \geq\left(1-2^{-4}\right) \sum_{w_{\nu} \notin \Delta(z)} a\left(z, w_{\nu}\right) \\
& \geq \frac{9}{10} \sum_{w_{\nu} \notin \Delta(z)} a\left(z, w_{\nu}\right) .
\end{aligned}
$$


Therefore, if $z$ satisfies (3.15) with $\lambda \geq 1 / 2$,

$$
\begin{aligned}
& \left(1-|z|^{2}\right)\left|\frac{\left(u_{p} B_{p}\right)^{\prime}(z)}{\left(u_{p} B_{p}\right)(z)}\right| \\
& \stackrel{\text { by (3.14) }}{\geq}\left|\sum_{w_{\nu} \notin \Delta(z)} a\left(z, w_{\nu}\right)\left(\frac{1-w_{\nu} \bar{z}}{z-w_{\nu}}\right)\right|-\sum_{w_{\nu} \in \Delta(z)} a\left(z, w_{\nu}\right) \rho\left(z, w_{\nu}\right)^{-1}
\end{aligned}
$$$$
\stackrel{\text { by (3.18) }}{\geq} \frac{9}{10} \sum_{w_{\nu} \notin \Delta(z)} a\left(z, w_{\nu}\right)-\frac{1}{\lambda} \sum_{w_{v} \in \Delta(z)} a\left(z, w_{\nu}\right)
$$

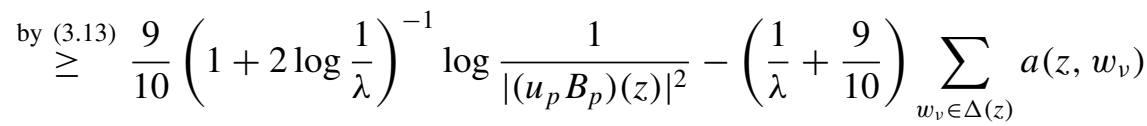$$
\stackrel{\text { for }}{\lambda \geq 1 / 2} \frac{9}{30} \log \frac{1}{\left|\left(u_{p} B_{p}\right)(z)\right|^{2}}-3 \sum_{w_{\nu} \in \Delta(z)} a\left(z, w_{\nu}\right)
$$$$
\stackrel{\text { by (3.17) }}{\geq} 3\left\{\frac{2}{10} \log \frac{1}{\left|\left(u_{p} B_{p}\right)(z)\right|}-\left(\frac{C_{1}}{\delta M}+C^{\prime}(\varrho, \alpha, M)\right)\right\} \text {. }
$$

Let us assume in addition to (3.15) that $\log \frac{1}{\left|\left(u_{p} B_{p}\right)(z)\right|}=C_{2} / 2 \delta$, where $C_{2}$ is the constant of (3.11). So, $\left|\left(u_{p} B_{p}\right)(z)\right|=e^{-C_{2} / 2 \delta}$ and

$$
\begin{aligned}
\left(1-|z|^{2}\right)\left|\left(u_{p} B_{p}\right)^{\prime}(z)\right| & \geq 3 e^{-C_{2} / 2 \delta}\left\{\frac{2}{10} \frac{C_{2}}{2 \delta}-\left(\frac{C_{1}}{\delta M}+C^{\prime}(\varrho, \alpha, M)\right)\right\} \\
& =3 e^{-C_{2} / 2 \delta}\left\{\frac{1}{\delta}\left(\frac{C_{2}}{10}-\frac{C_{1}}{M}\right)-C^{\prime}(\varrho, \alpha, M)\right\} .
\end{aligned}
$$

Now we fix $M$ big enough so that $\frac{C_{2}}{10}-\frac{C_{1}}{M}>\frac{C_{2}}{20}$. Since $M$ can be chosen to be an absolute constant, so does $\varrho=\varrho(M)$ and $\lambda$. Since $\alpha$ depended only on $\varrho$ and $\beta$ for (3.9) to hold, at this point we have that $\alpha=\alpha(\beta)$. So, actually $C^{\prime}(\varrho, \alpha, M)=C^{\prime}(\beta)$. We should mention that $C^{\prime}$ could be very big if so is $M$, but in any case, $C^{\prime}$ depends only on $\beta$ once we have chosen the absolute constant $M$. Therefore there is some $\delta_{\beta}$ small enough such that for any $\delta<\delta_{\beta}$ the expression between keys is positive. Hence,

$$
\left(1-|z|^{2}\right)\left|\left(u_{p} B_{p}\right)^{\prime}(z)\right|>3 e^{-C_{2} / 2 \delta}\left\{\frac{1}{\delta}\left(\frac{C_{2}}{20}\right)-C^{\prime}(\beta)\right\}=\tau(\delta, \beta)>0
$$

when $\delta<\delta_{\beta}, h\left(z, \Lambda_{p}\right) \geq \varrho$ and $\left|\left(u_{p} B_{p}\right)(z)\right|=e^{-C_{2} / 2 \delta}$.

Let $\omega$ be any point with $|\omega|=e^{-C_{2} / 2 \delta}$ and consider $A_{\omega}=\left(u_{p} B_{p}-\omega\right) /(1-$ $\left.\bar{\omega} u_{p} B_{p}\right)$. By Frostman's theorem [8], $A_{\omega}$ is a Blaschke product for almost 
every point $\omega$ in this circle. If $A_{\omega}$ is a Blaschke product factorize $A_{\omega}=C_{\omega} D_{\omega}$, where the zeros of $C_{\omega}$ are those zeros of $A_{\omega}$ whose hyperbolic distance to $\Lambda_{p}$ is at least $\varrho$ (i.e.: condition (3.15)), and the zeros of $D_{\omega}$ are the zeros $z$ of $A_{\omega}$ for which

$$
\inf _{\xi \in \Lambda_{p}} h(z, \xi)<\varrho .
$$

If $z$ is a zero of $C_{\omega}$ then by (3.19),

$$
\begin{aligned}
\left(1-|z|^{2}\right)\left|C_{\omega}^{\prime}(z)\right| \geq\left(1-|z|^{2}\right) \mid & A_{\omega}^{\prime}(z) \mid \\
& =\frac{\left(1-|z|^{2}\right)}{\left(1-|\omega|^{2}\right)}\left|\left(u_{p} B_{p}\right)^{\prime}(z)\right| \geq \frac{\tau(\delta, \beta)}{1-e^{-C_{2} / \delta}} .
\end{aligned}
$$

Consequently, $C_{\omega}$ is an interpolating Blaschke product and $\left\|\mu_{C_{\omega}}\right\|_{c} \leq k=$ $k(\delta, \beta)$ (see $[10, \mathrm{VII}])$. On the other hand, if $z$ is a zero of $D_{\omega}$, condition (3.20) says that there is some $z^{*} \in \Lambda_{p}$ such that $h\left(z, z^{*}\right)<\varrho$. But on $\Lambda_{p}$ we have

$$
\left|D_{\omega}\right| \geq\left|A_{\omega}\right|=\left|\frac{u_{p} B_{p}-\omega}{1-\bar{\omega} u_{p} B_{p}}\right| \geq \frac{|\omega|-\left|B_{p}\right|}{2} \geq \frac{e^{-\frac{C_{2}}{2 \delta}}-e^{-\frac{C_{2}}{\delta}}}{2}=\varphi(\delta)>0
$$

where the third inequality follows from (3.11). So, $\left|D_{\omega}\left(z^{*}\right)\right| \geq \varphi(\delta)$ and consequently Corollary 2.3 says that $\left\|\mu_{D_{\omega}}\right\|_{c} \leq C=C(\varrho, \delta)$. Since we have chosen $\varrho$ as an absolute constant, then the last constant only depends on $\delta$. Summing up, $\left\|\mu_{A_{\omega}}\right\| \leq\left\|\mu_{C_{\omega}}\right\|+\left\|\mu_{D_{\omega}}\right\| \leq K=K(\delta, \beta)$.

If $|\omega|=e^{-C_{2} / 2 \delta}$ is such that $A_{\omega}$ is a Blaschke product, we rename $A_{\omega}=B_{p}^{*}$. We are going to prove a lower bound for $\left|B_{p}\right|$ when $|u| \geq \beta$. If $|u(z)| \geq \beta$ then (3.10) says that $h\left(z, \Lambda_{p}\right) \geq \varrho$, that is, condition (3.15) holds. So, if as before, we denote $\Delta(z)=\{\omega \in \mathrm{D}:|z-\omega|<A(1-|z|)\}$ and $z_{v}$ are the zeros of $B_{p}$, (3.17) already gave us

$$
\sum_{z_{\nu} \in \Delta(z)} a\left(z, z_{\nu}\right) \leq \frac{C_{1}}{\delta M}
$$

since the constant $C^{\prime}(\varrho, \alpha, M)$ appearing in (3.17) comes from $u_{p}$, which is absent here. Also, we have by (3.12) that

$$
\sum_{z_{v} \notin \Delta(z)} a\left(z, z_{v}\right) \leq \frac{C_{2}}{20 \delta} .
$$

Thus, as in (3.13), we have

$$
\log \frac{1}{\left|B_{p}(z)\right|^{2}} \leq\left(1+2 \log \frac{1}{\lambda}\right) \sum_{\nu} a\left(z, z_{v}\right) \leq\left(1+2 \log \frac{1}{\lambda}\right)\left(\frac{C_{1}}{\delta M}+\frac{C_{2}}{20 \delta}\right) .
$$


Since we have chosen $\varrho$ so that $\lambda=\lambda(\varrho)>1 / 2$ then $\log \lambda^{-1}<1$, and since $M$ was chosen so that $\frac{C_{2}}{10}-\frac{C_{1}}{M}>\frac{C_{2}}{20}$, then $\frac{C_{1}}{M}<\frac{C_{2}}{20}$. Hence,

$$
\log \left|B_{p}(z)\right|^{-1} \leq \frac{3 C_{2}}{2 \delta}\left(\frac{1}{20}+\frac{1}{20}\right) \leq \frac{3}{20} \frac{C_{2}}{\delta},
$$

or equivalently, $\left|B_{p}(z)\right|^{-1} \leq \exp \left(\frac{3 C_{2}}{20 \delta}\right)$. Consequently, on the set where $|u| \geq \beta$ we have

$\left|u_{p}-\frac{B_{p}^{*}}{B_{p}}\right|=\frac{1}{\left|B_{p}\right|}\left|\frac{\bar{w}\left(B_{p} u_{p}\right)^{2}-w}{1-\bar{w} B_{p} u_{p}}\right| \leq \frac{2|w|}{\left|B_{p}\right|(1-|w|)} \leq \frac{2 e^{-7 C_{2} / 20 \delta}}{1-e^{-C_{2} / 2 \delta}}=\phi(\delta)$,

where $\phi(\delta) \rightarrow 0$ when $\delta \rightarrow 0$. Finally, the above inequalities easily show that $B_{p}^{*}$ satisfies condition (c) of the lemma.

THEOREM 3.3. Let $u$ be an inner function. Given constants $0<\beta<1$ and $\varepsilon>0$, there exist two Carleson-Newman Blaschke products $B_{1}$ and $B_{2}$ such that

(a) $\left|u(z)-B_{1}(z) / B_{2}(z)\right|<\varepsilon$ if $|u(z)|>\beta$,

(b) $\left\|\mu_{B_{1}}\right\|_{c},\left\|\mu_{B_{2}}\right\|_{c} \leq c_{1}(\varepsilon, \beta)$,

(c) $\left|B_{1}(z)\right| \geq c_{2}(\varepsilon, \beta)$ and $\left|B_{2}(z)\right| \geq c_{2}(\varepsilon, \beta)$ if $|u(z)|>\beta$,

where $c_{1}(\varepsilon, \beta)$ and $c_{2}(\varepsilon, \beta)$ are positive constants only depending on $\varepsilon$ and $\beta$.

Proof. By Frostman's theorem [8] $u$ can be uniformly approximated by Blaschke products, so we can assume that $u$ is a Blaschke product.

Observe that since the integer $M$ in the previous lemma is an absolute constant, the theorem follows immediately by choosing $B_{1}=u_{0} \prod_{p=1}^{M} B_{p}$ and $B_{2}=\prod_{p=1}^{M} B_{p}^{*}$. The constant $c_{1}$ here is $M+1$ times the corresponding constant in the lemma, and $c_{2}$ here is $\beta$ times the $M$-th power of the corresponding constant in the lemma.

The origin of the above theorem can be traced to the Douglas-Rudin Theorem, which states that every function whose modulus is 1 almost everywhere on $\partial \mathrm{D}$ can be approximated in $L^{\infty}(\partial \mathrm{D})$-norm by quotients of Blaschke products [6]. Later, in [12], Jones proved that the Blaschke products can be chosen to be interpolating, with the additional kind of control given by item (b) of the theorem. However, in his construction the zeros of $B_{1}$ and $B_{2}$ can be located in regions where $|u|$ is close to 1 . In [15] the second author proved that there are interpolating Blaschke products satisfying (a), but without obtaining the control on the constants given by (b) and (c). That is, Theorem 3.3 merges the main features of the approximation results given in [12] and [15]. 


\section{The proofs of Theorems $A$ and $B$}

If $B$ is a Blaschke product we write $Z(B)$ for the sequence of its zeros, and if $a \in Z(B)$, let $B_{a}$ be the Blaschke product resulting from removing from $B$ one factor

$$
\phi_{a}(z)=\frac{\bar{a}}{a} \frac{(a-z)}{(1-\bar{a} z)} \quad(a \neq 0),
$$

and $\phi_{a}(z)=z$ if $a=0$. Denote

$$
\delta(B):=\inf \left\{\left(1-|a|^{2}\right)\left|B^{\prime}(a)\right|: a \in Z(B)\right\}=\inf \left\{\left|B_{a}(a)\right|: a \in Z(B)\right\} .
$$

By Carleson's theorem [2] a Blaschke product $B$ is interpolating if and only if $\delta(B)>0$. It is easy to prove and well known that if $\delta(B)>0$ then $\mu_{B}$ is a Carleson measure of intensity bounded by a constant depending on $\delta(B)$.

Lemma 4.1. Let B be a Blaschke product such that $\mu_{B}$ is a Carleson measure with intensity bounded by $M$. Let $g \in H^{\infty}$, with $\|g\| \leq 1$, and assume that

$$
|B(z)|>\gamma \quad \text { if } \quad|g(z)|<\delta,
$$

where $\gamma$ and $\delta$ are fixed constants between 0 and 1 . Then for any $\varepsilon>0$ there exists a function $H \in H^{\infty}$ and an absolute constant $v>1$ such that

$$
|B(z)-H(z)|<\varepsilon \quad \text { if } \quad|g(z)|<\delta^{\nu}
$$

and $C \leq|H(z)| \leq C^{-1}$, where $C=C(M, \gamma, \delta, \varepsilon)$.

Proof. Since $\mu_{B}=\sum\left(1-\left|z_{n}\right|\right) \delta_{z_{n}}$ is a Carleson measure then $B$ is a finite product of interpolating Blaschke products. More quantitatively, if the Carleson intensity of $\mu_{B}$ is bounded by $M$, given $0<\beta<1$, one can decompose the Blaschke product $B$ into at most $k_{0}=k_{0}(M, \beta)$ factors

$$
B=B_{1} \ldots B_{k_{0}}
$$

such that $\delta\left(B_{i}\right) \geq \beta$ for $i=1, \ldots, k_{0}$. Indeed, by taking $[M]+1$ if necessary, we can assume that $M$ is an integer. Thus, if $Q \subset \mathrm{D}$ is any angular square lying on $\partial \mathrm{D}$, the top half of $Q$ contains at most $2 M$ points of $\left\{z_{n}\right\}$. It is then known (see the construction in [13, pp. 158-159]) that $\left\{z_{n}\right\}$ splits into at most $8 M$ sequences $\left\{z_{n}^{(j)}\right\}, j=1, \ldots, m \leq 8 M$, such that for each $j$,

$$
\rho\left(z_{n}^{(j)}, z_{k}^{(j)}\right) \geq 1 / 5 \quad \text { for } n \neq k .
$$

Let $A_{j}$ be the Blaschke product with zeros $\left\{z_{n}^{(j)}\right\}(1 \leq j \leq m)$. By [10, VII, Thm. 1.1] there is $\delta_{0}=\delta_{0}(M)>0$, such that $\delta\left(A_{j}\right) \geq \delta_{0}$ for all $j$. Our 
claimed factorization follows from the fact that each $A_{j}$ can be factorized as $A_{j}=A_{j}^{1} A_{j}^{2}$, with $\delta\left(A_{j}^{i}\right) \geq \delta\left(A_{j}\right)^{1 / 2} \geq \delta_{0}^{1 / 2}$ (see [10, X, Cor. 1.6]). Hence, fixed $0<\beta<1$, it is sufficient to prove the lemma for each subfactor $B_{i}$, $i=1, \ldots, k_{0}$. That is, we may assume that $\delta(B) \geq \beta$, where $0<\beta<1$ is an absolute constant to be determined later.

Let $\{Q\}$ be the decomposition of the unit disk into dyadic angular squares of the form

$$
Q=\left\{r e^{i \theta}: \frac{2 \pi j}{2^{k}} \leq \theta<\frac{2 \pi(j+1)}{2^{k}}, 0<1-r \leq 2^{-k}\right\},
$$

$j=0, \ldots, 2^{k}-1, k \geq 0$. Observe that the pseudohyperbolic diameter of

$$
T(Q)=\left\{r e^{i \theta} \in Q: 2^{-k-1}<1-r \leq 2^{-k}\right\}
$$

is bounded by an absolute constant $\alpha_{0}<1$. We pick the dyadic squares $Q$ containing a zero, say $a$, of $B$ in its top part and rename them as $\left\{Q_{a}\right.$ : $B(a)=0\}$. Since $\left|B_{a}(a)\right|>\beta$ for all $a \in Z(B)$, by Schwarz's Lemma $\left|B_{a}(z)\right|>\left(\beta-\alpha_{0}\right) /\left(1-\beta \alpha_{0}\right)$ for any $z \in T\left(Q_{a}\right)$. So, if $\beta=\beta\left(\alpha_{0}\right)$ is close enough to 1 , we can assume that

$$
\inf \left\{\left|B_{a}(z)\right|: z \in T\left(Q_{a}\right)\right\}>\alpha_{0}^{1 / 2}
$$

for every $a \in Z(B)$. Hence, each $T(Q)$ contains at most one single zero of $B$. If $a, b$ are distinct zeros of $B$, the inequalities $\rho(a, b) \geq\left|B_{a}(a)\right| \geq \beta$ imply that

$$
\rho\left(T\left(Q_{a}\right), T\left(Q_{b}\right)\right) \rightarrow 1 \quad \text { when } \quad \beta \rightarrow 1 .
$$

Therefore, if $\beta=\beta\left(\alpha_{0}\right)$ is close enough to 1 , we have

$$
\rho\left(T\left(Q_{a}\right), T\left(Q_{b}\right)\right) \geq \alpha_{0}^{1 / 2} .
$$

For each zero $a$ of $B$ we will construct a slit $\Gamma_{a}=H_{a} \cup V_{a}$ consisting of an angular $\operatorname{arc} H_{a}$ and a radial arc $V_{a}$ such that

$$
\inf \left\{\left|B_{a}(z)\right|: z \in \Gamma_{a}\right\} \geq \alpha_{0}^{1 / 2} .
$$

Since $\left|B_{a}(a)\right| \geq \beta$, a well known application of Hall's lemma (see [10, VIII]) to the function $B_{a}$ gives that if $\beta=\beta\left(\alpha_{0}^{1 / 2}\right)$ is chosen sufficiently close to 1 ,

$$
\mid\left\{e^{i \theta} \in \partial \mathrm{D}: r e^{i \theta} \in Q_{a} \text { with }\left|B_{a}\left(r e^{i \theta}\right)\right|<\alpha_{0}^{1 / 2}\right\} \mid<\frac{\ell\left(Q_{a}\right)}{2} .
$$

Consequently, there exists a radial line $V_{a}^{\prime}$ in $Q_{a}$ such that $\left|B_{a}(z)\right| \geq \alpha_{0}^{1 / 2}$ for any $z \in V_{a}^{\prime}$. We consider the angular arc $H_{a}$ from the point $a$ to $V_{a}^{\prime}$ and the 
portion $V_{a}$ of $V_{a}^{\prime}$ that goes from $H_{a}$ to the unit circle. Write $\Gamma_{a}=H_{a} \cup V_{a}$. So, (4.3) holds by construction and (4.1). See Figure 3.

Let $a$ and $b$ be different zeros of $B$. Observe that since by (4.3), $\rho(b, z) \geq$ $\left|B_{a}(z)\right| \geq \alpha_{0}^{1 / 2}$ for any $z \in V_{a}$, then $\rho\left(b, V_{a}\right) \geq \alpha_{0}^{1 / 2}$. Hence $\rho\left(T\left(Q_{b}\right), V_{a}\right) \geq$ $\left(\alpha_{0}^{1 / 2}-\alpha_{0}\right) /\left(1-\alpha_{0} \alpha_{0}^{1 / 2}\right)$. So, if $|a| \leq|b|$,

$$
\rho\left(Q_{b}, V_{a}\right)=\rho\left(T\left(Q_{b}\right), V_{a}\right) \geq \frac{\alpha_{0}^{1 / 2}-\alpha_{0}}{1-\alpha_{0} \alpha_{0}^{1 / 2}},
$$

while by (4.2),

$$
\rho\left(H_{a}, H_{b}\right) \geq \rho\left(T\left(Q_{a}\right), T\left(Q_{b}\right)\right) \geq \alpha_{0}^{1 / 2}
$$

and

$$
\rho\left(H_{a}, V_{b}\right) \geq \rho\left(T\left(Q_{a}\right), V_{b}\right) \geq \frac{\alpha_{0}^{1 / 2}-\alpha_{0}}{1-\alpha_{0} \alpha_{0}^{1 / 2}} .
$$

Thus, in addition to (4.3), the slits $\left\{\Gamma_{a}: a \in Z(B)\right\}$ satisfy

$$
\rho\left(\Gamma_{a}, \Gamma_{b}\right) \geq \alpha_{1}:=\frac{\alpha_{0}^{1 / 2}-\alpha_{0}}{1-\alpha_{0} \alpha_{0}^{1 / 2}} .
$$

Also, observe that since $\alpha_{0}$ is fixed at the beginning as an absolute constant, so are $\beta=\beta\left(\alpha_{0}\right)$ and $\alpha_{1}=\alpha_{1}\left(\alpha_{0}\right)$. For a fixed $\tau=\tau(\gamma)<\min \left\{\alpha_{1} / 4, \gamma / 2\right\}$, let us consider

$$
\tilde{\Gamma}_{a}=\left\{z \in \mathrm{D}: \rho\left(z, \Gamma_{a}\right) \leq \tau\right\} .
$$

The region $\tilde{\Gamma}_{a}$ looks like a conical neighborhood of the slit $\Gamma_{a}$. Since $\tau<\alpha_{1} / 4$, (4.4) implies that $\tilde{\Gamma}_{a} \cap \tilde{\Gamma}_{b}=\emptyset$ whenever $a$ and $b$ are two different zeros of $B$. Also, since $\tau<\gamma / 2$, the hypothesis that $|B(z)|>\gamma$ when $|g(z)|<\delta$ yields

$$
\{z: \rho(z, Z(B)) \leq \tau\} \cap\{z:|g(z)|<\delta\}=\emptyset .
$$

For each zero $a$ of $B$ consider a branch of $\log \varphi_{a}(z)$ defined in $D \backslash \Gamma_{a}$ that jumps $2 \pi i$ when $z$ crosses $\Gamma_{a} \backslash\{a\}$. By regularization we can obtain a smooth function $\psi_{a}$ on D with $\psi_{a} \equiv \log \varphi_{a}$ in $\mathrm{D} \backslash \tilde{\Gamma}_{a}, \operatorname{Re} \psi_{a} \equiv \log \left|\varphi_{a}\right|$ in $\{z: \rho(z, a) \geq \tau / 4\}$, $\left|\psi_{a}\right| \leq C$, and

$$
\begin{aligned}
& 0 \leq \operatorname{Im} \psi_{a} \leq 4 \pi \\
& (1-|z|)\left|\bar{\partial} \psi_{a}(z)\right| \leq C, \\
& (1-|z|)^{2}\left|\Delta \psi_{a}(z)\right| \leq C,
\end{aligned}
$$


where $C=C(\tau)$ is an absolute constant only depending on $\tau$. The Blaschke condition implies that the sum $\psi(z):=\sum_{a} \psi_{a}(z)$ converges uniformly on compact sets of the unit disk. Observe that $|\operatorname{Re} \psi(z)| \leq C(\beta)$ for any $z \in \mathrm{D}$.

By Lemma 2.1 there is an absolute constant $v_{0}>1$ such that if $Q$ is an angular square and any of the sets

$$
\left\{r: \exists r e^{i t} \in Q,\left|g\left(r e^{i t}\right)\right|<\delta^{\nu_{0}}\right\}, \quad\left\{e^{i t}: \exists r e^{i t} \in Q,\left|g\left(r e^{i t}\right)\right|<\delta^{\nu_{0}}\right\},
$$

has length bigger than $\ell(Q) / 8$ then

$$
|g(z)|<\delta \quad \text { on } \quad\{z \in Q: 1-|z| \geq \ell(Q) / 4\} .
$$

Let $\Omega$ be any connected component of $\left\{z:|g(z)|<\delta^{\nu_{0}}\right\}$ (showed in Figure 3). Claim: the number of slits $\Gamma_{a}, a \in Z(B)$, which meet $\Omega$ is bounded by a constant $C=C(\tau)$ (independent of $\Omega$ ). To prove the Claim let $Q$ be a minimal angular square containing $\Omega$, and observe that if $a \in Z(B), \ell\left(Q_{a}\right) \geq \ell(Q) / 10$ and $\Gamma_{a} \cap \Omega \neq \emptyset$, then $\Gamma_{a}$ must meet $\{z \in Q: 1-|z|<\ell(Q) / 10\}$. Since this set has bounded hyperbolic diameter, (4.4) implies that the number of such zeros is bounded by a constant $C(\tau)$. On the other hand, if $a \in Z(B)$ with $1-|a|<\ell(Q) / 10$, an argument of Treil [16] will show that $\Gamma_{a}$ cannot meet $\Omega$. Indeed, if $\Gamma_{a} \cap \Omega \neq \emptyset$, taking the angular square $R_{a}$ whose base has the same center as $Q_{a}$ and twice its length, the angular or the radial projection of $R_{a} \cap \Omega$ must have length $\geq \ell\left(R_{a}\right) / 4$. Hence, (4.8) says that $|g(z)|<\delta$ on $\left\{z \in R_{a}: 1-|z| \geq \ell(Q) / 4\right\}$. Consequently $|g(a)|<\delta$, which is a contradiction because $a$ is a zero of $B$.

Fix $z_{0} \in \Omega$ and let $\log B$ be a suitable branch of the logarithm of $B$ on $\Omega$ with $\log B\left(z_{0}\right)=\psi\left(z_{0}\right)$. Since (4.5) implies that $\left|\log \varphi_{a}-\psi_{a}\right| \leq 4 \pi$ on $\left\{|g|<\delta^{\nu_{0}}\right\}$ and $\Omega$ is an arbitrary component, the above Claim proves that

$$
|\log B(z)-\psi(z)| \leq C_{1}(\tau) \quad \text { if }|g(z)|<\delta^{\nu_{0}} .
$$

Next, consider the equation $\bar{\partial} b=\bar{\partial} \psi$. Since $\left\{\tilde{\Gamma}_{a}: a \in Z(B)\right\}$ are pairwise disjoint, it follows from (4.6) and (4.7) that

$$
\begin{aligned}
(1-|z|)|\bar{\partial} \psi(z)| & \leq C=C(\tau), \\
(1-|z|)^{2}|\Delta \psi(z)| & \leq C=C(\tau) .
\end{aligned}
$$

Also, since the support of $\bar{\partial} \psi$ is contained in $\cup \tilde{\Gamma}_{a}$ and $\sum_{Z(B)}(1-|a|) \delta_{a}$ is a Carleson measure with intensity bounded by a constant depending only on $\beta$, then $|\bar{\partial} \psi(z)| d m(z)$ and $|\Delta \psi(z)|(1-|z|) d m(z)$ are Carleson measures with intensity bounded by $C(\beta, \tau)$. So, applying Lemma 2.5 , we obtain a function 


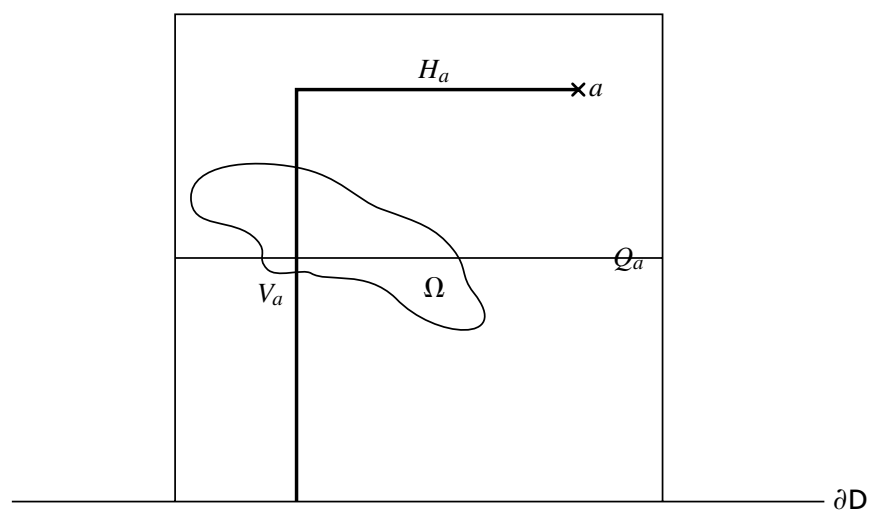

FIGURE 3.

$b$ with $\bar{\partial} b=\bar{\partial} \psi$ such that

$$
\sup \{|b(z)|: z \in \mathrm{D}\} \leq C^{\prime}(\beta, \tau) .
$$

Hence, the function $h=\psi-b$ is analytic, and by (4.9) satisfies

$$
|\log B(z)-h(z)| \leq C_{1}(\beta, \tau) \quad \text { if } \quad|g(z)|<\delta^{\nu_{0}} .
$$

Observe also that

$$
\sup _{\mathrm{D}}|\operatorname{Re} h(z)| \leq \sup _{\mathrm{D}}|\operatorname{Re} \psi(z)|+\|b\|_{\infty}<C_{2}(\beta, \tau) .
$$

Applying Theorem 3.1, for any $\varepsilon>0$ there exists a function $G=G_{\varepsilon} \in H^{\infty}$ with $\|G\|_{\infty}<C_{3}=C_{3}(\beta, \tau, \delta, \varepsilon)$, such that

$$
|\log B(z)-h(z)-G(z)| \leq \varepsilon \quad \text { if } \quad|g(z)|<\delta^{\nu_{0}+1} .
$$

At this point we can choose $v=v_{0}+1$, and recalling that $\tau$ depends only on $\gamma$, and $\beta$ is an absolute constant, we actually have that the dependency of the above constant is $C_{3}=C_{3}(\gamma, \delta, \varepsilon)$. Therefore, using the basic inequality $\left|e^{z}-1\right| \leq|z| e^{|z|}$, if $|g(z)|<\delta^{\nu}$ we have

$$
\begin{aligned}
|B(z)-\exp (h(z)+G(z))| & \leq \varepsilon \exp [\varepsilon+\operatorname{Re}(h(z)+G(z))] \\
& \leq \varepsilon \exp (2 \varepsilon+|\log | B(z)||) \leq \frac{\varepsilon e^{2 \varepsilon}}{\gamma} .
\end{aligned}
$$

So, taking $H=\exp (h+G)$ completes the proof. 
Lemma 4.2. Let $f \in H^{\infty}$ with $\|f\| \leq 1$. Given $\varepsilon>0$ and $0<\gamma<1$, there is a Blaschke product $B$ and an invertible function $G \in\left(H^{\infty}\right)^{-1}$ such that

(a) $\|f-G B\|<\varepsilon$,

(b) $1 \geq|G| \geq c(\varepsilon)$ on $\mathrm{D}$, where $c(\varepsilon)>0$ depends only on $\varepsilon$,

(c) $|B|>\gamma / 2$ when $|f|>\gamma$.

Proof. First observe that by restricting $\varepsilon<\gamma / 2$, (a) and (b) imply (c), since when $|f|>\gamma$,

$$
|B| \geq|G B| \geq|f|-|f-G B|>\gamma-\varepsilon>\gamma / 2 .
$$

Let $f=f_{0} v$ be the outer-inner factorization of $f$, and factorize further $f_{0}=$ $f_{1} f_{2}$, where $f_{j}(j=1,2)$ are outer functions such that on $\partial \mathrm{D}$,

$$
\left|f_{1}\right|= \begin{cases}|f| & \text { if } \varepsilon / 2<|f| \leq 1 \\ 1 & \text { if }|f| \leq \varepsilon / 2\end{cases}
$$

and

$$
\left|f_{2}\right|= \begin{cases}1 & \text { if } \varepsilon / 2<|f| \leq 1 \\ |f| & \text { if }|f| \leq \varepsilon / 2\end{cases}
$$

Hence, for almost every point of the unit circle we have, $\varepsilon / 2 \leq\left|f_{1}\right| \leq 1$, and $0 \leq\left|f_{2}\right| \leq \varepsilon / 2$ or $\left|f_{2}\right|=1$. If $f_{\varepsilon}=\left(f_{2}-\varepsilon\right) /\left(1-\varepsilon f_{2}\right)$ then

$$
\left|f_{\varepsilon}\right| \geq \frac{\varepsilon / 2}{\left|1-\varepsilon f_{2}\right|} \geq \frac{\varepsilon}{4} \quad \text { a.e. on } \quad \partial \mathrm{D} \text {. }
$$

Now let $f_{1} f_{\varepsilon}=G u$, with $G$ outer and $u$ inner. Then $|G|=\left|f_{1} f_{\varepsilon}\right| \geq \varepsilon^{2} / 8$ a.e. on $\partial \mathrm{D}$, and since $G$ is outer, also on D. Additionally,

$$
\begin{aligned}
|f-G u v| & =\left|f_{1} f_{2} v-f_{1} f_{\varepsilon} v\right|=\left|f_{1} v\right|\left|f_{2}-f_{\varepsilon}\right| \\
& =\left|f_{1} v\right| \varepsilon\left|\frac{1-f_{2}^{2}}{1-\varepsilon f_{2}}\right| \leq \frac{2 \varepsilon}{1-\varepsilon} \leq 4 \varepsilon,
\end{aligned}
$$

where the last inequality comes from $\varepsilon<\gamma / 2<1 / 2$. Since every inner function can be uniformly approximated by Blaschke products, we can approximate $u v$ by a Blaschke product $B$ so that (a) follows.

Lemma 4.3. Let $f, g \in H^{\infty}$, with $\|g\| \leq 1$, such that

$$
|f(z)|>\gamma \quad \text { if } \quad|g(z)|<\delta .
$$


Then given $\varepsilon>0$ there exists $F \in H^{\infty}$ such that

(a) $|f(z)-F(z)|<\varepsilon$ if $|g(z)|<\delta^{v}$, where $v$ is the absolute constant of Lemma 4.1,

(b) $C^{-1}<|F(z)|<C$, for any point $z \in \mathrm{D}$, where $C=C(\|f\|, \gamma, \delta, \varepsilon)$.

Proof. There is no loss of generality if we assume $\|f\| \leq 1$. By Lemma 4.2 there are $G \in H^{\infty}$ and a Blaschke product $B$ such that $c(\varepsilon)<|G|<1$ on D, $|B|>\gamma / 2$ on $\{|g|<\delta\}$, and

$$
\|f-G B\|<\varepsilon .
$$

By Theorem 3.3 there are Carleson-Newman Blaschke products $B_{1}$ and $B_{2}$, with the intensities of the Carleson measures $\mu_{B_{1}}$ and $\mu_{B_{2}}$ bounded by $M=$ $M(\varepsilon, \gamma)$, such that

$$
\left|B-B_{1} / B_{2}\right|<\varepsilon \quad \text { and } \quad\left|B_{j}\right|>c_{2}=c_{2}(\varepsilon, \gamma / 4) \quad \text { if } \quad|B|>\gamma / 4
$$

for $j=1,2$. Let $\varepsilon_{1}<c_{2} / 2$ to be chosen later. By Lemma 4.1 there are $H_{j} \in H^{\infty}$ for $j=1,2$ such that $\left|B_{j}-H_{j}\right|<\varepsilon_{1}$ if $|g|<\delta^{\nu}$ and $C^{-1}<\left|H_{j}\right|<$ $C$ on $\mathrm{D}$, with $C=C\left(M, c_{2}, \delta, \varepsilon_{1}\right)=C\left(\gamma, \delta, \varepsilon_{1}\right)$. Therefore the function $F=G H_{1} H_{2}^{-1}$ satisfies condition (b) of the lemma. Besides, when $|g|<\delta^{\nu}$,

$$
\left|H_{2}\right| \geq\left|B_{2}\right|-\left|H_{2}-B_{2}\right|>c_{2}-\varepsilon_{1}>c_{2} / 2,
$$

and consequently

$$
\begin{aligned}
\left|H_{1} H_{2}^{-1}-B_{1} B_{2}^{-1}\right| & \leq\left|H_{2}^{-1}\right|\left|H_{1}-B_{1}\right|+\left|B_{1}\right|\left|H_{2}^{-1}-B_{2}^{-1}\right| \\
& \leq \frac{2 \varepsilon_{1}}{c_{2}}+\frac{2 \varepsilon_{1}}{c_{2}^{2}}<\varepsilon
\end{aligned}
$$

if we choose $\varepsilon_{1}=\varepsilon_{1}(\varepsilon, \gamma)$ small enough. Hence, (4.12), (4.11) and (4.10) say that when $|g|<\delta^{v}$,

$$
|F-f| \leq\left|G H_{1} H_{2}^{-1}-G B_{1} B_{2}^{-1}\right|+\left|G B_{1} B_{2}^{-1}-G B\right|+|G B-f|<3 \varepsilon,
$$

as claimed.

Proof of Theorem B. The purpose of the theorem is to remove the restriction $|g|<\delta^{v}$ from (a) of Lemma 4.3, and replace it by $|g|<\eta$ for an arbitrary $\eta \in(0, \delta)$. If $\delta \geq \eta^{1 / v}$ the result follows immediately from Lemma 4.3. So, the strategy of the proof consists on iterating a process of approximation in order to arrive to a situation in which the above inequality holds. If $\delta<\eta^{1 / v}$ consider the parameter

$$
r=\left(\frac{\delta^{\nu}}{\eta}\right)^{\frac{1}{v-1}} .
$$


Then $r<1$ and solving from $\delta>\eta$ yields $r>\delta$. Observe also that $\left(\frac{\delta}{r}\right)^{v}=\frac{\eta}{r}$. Let $g_{1}=g / r$ and

$$
\Omega=\{z \in \mathrm{D}:|g|<r\}=\left\{z \in \mathrm{D}:\left|g_{1}\right|<1\right\} .
$$

If $\mathscr{O} \subset \Omega$ is a connected component of $\Omega$, then it is conformally equivalent to $D$, and by restriction, we can think of the functions in $H^{\infty}$ as functions of $H^{\infty}(\mathscr{O})$. Thus, $\left.g_{1}\right|_{\mathcal{O}} \in H^{\infty}(\mathcal{O})$ has norm 1. Since $|f|_{\mathscr{O}} \mid>\gamma$ when $\left|g_{1}\right|_{\mathscr{O}} \mid<\delta / r$, Lemma 4.3 says that there is $F_{\mathscr{O}} \in H^{\infty}(\mathscr{O})$ such that $\left|F_{\mathscr{O}}-f\right|_{\mathscr{O}} \mid<\varepsilon$ on $\left\{z \in \mathcal{O}:\left|g_{1}(z)\right|<(\delta / r)^{\nu}\right\}$ and $C_{0}<\left|F_{\mathcal{O}}\right|<C_{0}^{-1}$, where $C_{0}>0$ depends on $K, \gamma, \delta, \eta$ and $\varepsilon$. Since this holds for every component $\mathcal{O}$ of $\Omega$, we actually get $F \in H^{\infty}(\Omega)$ so that

$$
|F-f|_{\Omega} \mid<\varepsilon \quad \text { on } \quad\left\{\left|g_{1}\right|<(\delta / r)^{v}=\eta / r\right\}=\{|g|<\eta\}
$$

and $C_{0}<|F|<C_{0}^{-1}$ on $\Omega$. Write $\alpha=\min \left\{C_{0} / 2, \varepsilon\right\}$. By Theorem 3.1 there is $F_{1} \in H^{\infty}$ such that $\left|F_{1}-F\right|<\alpha$ on $\{|g|<(r+\delta) / 2\}$ and $\left\|F_{1}\right\| \leq K_{1}$, where $K_{1}$ is a constant depending on $C_{0}, r, \delta$ and $\alpha$. Then $\left|F_{1}\right| \geq C_{0} / 2$ on $\{|g|<(r+\delta) / 2\}$ and $\left|F_{1}-f\right|<2 \varepsilon$ on $\{|g|<\eta\}$.

We can iterate this process with $f$ replaced by $F_{1}, \gamma$ replaced by $C_{0} / 2$ and $\delta$ replaced by $\delta_{1}=(r+\delta) / 2$. At the $n$-step we will have:

$$
\delta_{n}=\frac{1}{2}\left[\left(\frac{\delta_{n-1}^{\nu}}{\eta}\right)^{\frac{1}{v-1}}+\delta_{n-1}\right]
$$

where $\delta_{0}=\delta$, and $F_{n} \in H^{\infty}$ such that

$$
\left|F_{n}-F_{n-1}\right|<2 \varepsilon \quad \text { on } \quad\{|g|<\eta\}, \quad\left(\text { with } F_{0}=f\right),
$$

$\left|F_{n}\right| \geq C_{n} / 2$ on $\left\{|g|<\delta_{n}\right\}$, and $\left\|F_{n}\right\| \leq K_{n}$, where $C_{n}, K_{n}>0$ depend on $n$, $K, \varepsilon, \gamma, \delta$ and $\eta$. Observe that (4.14) implies that $\left|F_{n}-f\right|<2 n \varepsilon$ on $\{|g|<\eta\}$. We can keep repeating this process as long as $\delta_{n}<1$. Indeed, if there is $n_{0}$ such that $\delta_{n_{0}} \geq 1$ for the first time, then

$$
\begin{aligned}
& \left|F_{n_{0}}-f\right|<2 n_{0} \varepsilon \quad \text { on } \quad\{|g|<\eta\}, \\
& \left|F_{n_{0}}\right| \geq C_{n_{0}} / 2 \quad \text { on } \quad\left\{|g|<\delta_{n_{0}}\right\}=\mathrm{D},
\end{aligned}
$$

and

$$
\left\|F_{n_{0}}\right\| \leq K_{n_{0}},
$$

where $C_{n_{0}}$ and $K_{n_{0}}$ only depend on the parameters stated in the lemma and $n_{0}$. Thus, the lemma will follow if we show that there is an integer $n_{0}$ depending only on $\delta$ and $\eta$ such that $\delta_{n_{0}} \geq 1$ for the first time. Clearly, it is enough to 
show that $\delta_{n} \rightarrow \infty$ as $n \rightarrow \infty$. We recall that $\delta>\eta$ implies $r=\left(\frac{\delta^{v}}{\eta}\right)^{\frac{1}{v-1}}>\delta$, and since $\delta_{1}$ is the average between $\delta$ and $r$ then $\delta_{1}>\delta=\delta_{0}$. The recursive definition of $\delta_{n}$ then gives that $\delta_{n}>\delta_{n-1}$. Therefore, this sequence either tends to infinity or has a finite positive limit. Suppose that $\lim \delta_{n}=\ell<\infty$. Then taking limits in (4.13) we obtain

$$
\ell=\frac{1}{2}\left[\left(\frac{\ell^{v}}{\eta}\right)^{\frac{1}{v-1}}+\ell\right],
$$

from which $\ell=0$ or $\eta$. But this is not possible because $\ell>\delta_{0}=\delta>\eta$.

LEMma 4.4. If $n$ is a positive integer, for $1 \leq j \leq n$ let $0<\eta_{j}<\delta_{j}<1$ and $f, g_{j} \in H^{\infty}$ such that $\left\|g_{j}\right\| \leq 1,\|f\| \leq K$ and $|f|>\gamma>0$ on the set $\cap_{j=1}^{n}\left\{\left|g_{j}\right|<\delta_{j}\right\}$. Then, given $\varepsilon>0$ there exists $F \in H^{\infty}$ such that

$$
|F-f|<\varepsilon \quad \text { on } \bigcap_{j=1}^{n}\left\{\left|g_{j}\right|<\eta_{j}\right\},
$$

and

$$
C_{n} \leq|F| \leq C_{n}^{-1},
$$

where $C_{n}>0$ is a constant independent of $f$ (it depends on $K, \gamma, \varepsilon, \delta_{j}, \eta_{j}$, $1 \leq j \leq n)$.

Proof. We will argue by induction on $n$. For $n=1$ the statement is precisely Theorem B. So suppose that the statement holds for $n-1$, and consider $\Omega=\left\{z \in \mathrm{D}:\left|g_{n}(z)\right|<\delta_{n}\right\}$. If $\mathscr{O} \subset \Omega$ is a connected component, then

$$
|f|_{\mathcal{O}} \mid>\gamma \quad \text { on } \quad \bigcap_{j=1}^{n-1}\left\{\left|g_{j}\right|<\delta_{j}\right\} \cap \mathcal{O} .
$$

By inductive hypothesis then there is $F_{\mathscr{O}} \in H^{\infty}(\mathscr{O})$ such that

$$
\left|F_{\mathscr{O}}-f\right|_{\mathscr{O}} \mid<\varepsilon \quad \text { on } \quad \bigcap_{j=1}^{n-1}\left\{\left|g_{j}\right|<\eta_{j}\right\} \cap \mathcal{O}
$$

and $C_{n-1} \leq\left|F_{\mathscr{O}}\right| \leq C_{n-1}^{-1}$ on $\mathcal{O}$, where the dependencies of $C_{n-1}$ are the ones stated in the lemma. Since this holds for every component $\mathcal{O} \subset \Omega$, we obtain a function $F_{0} \in H^{\infty}(\Omega)$ so that

$$
\left|F_{0}-f\right|_{\Omega} \mid<\varepsilon \quad \text { on } \quad \bigcap_{j=1}^{n-1}\left\{\left|g_{j}\right|<\eta_{j}\right\} \cap \Omega
$$


and $C_{n-1} \leq\left|F_{0}\right| \leq C_{n-1}^{-1}$ on $\Omega$. Since

$$
\Omega=\left\{\left|g_{n}\right|<\delta_{n}\right\} \supset\left\{\left|g_{n}\right|<\left(\delta_{n}+\eta_{n}\right) / 2\right\},
$$

Theorem 3.1 says that there exists $F_{1} \in H^{\infty}$ such that

$$
\left|F_{1}-F_{0}\right|<\alpha=\min \left\{C_{n-1} / 2, \varepsilon\right\} \quad \text { on } \quad\left\{\left|g_{n}\right|<\left(\delta_{n}+\eta_{n}\right) / 2\right\}
$$

and

$$
\left\|F_{1}\right\| \leq C\left(\eta_{n}, \delta_{n}, \alpha, C_{n-1}\right)
$$

Thus,

$$
\left|F_{1}\right| \geq\left|F_{0}\right|-\left|F_{0}-F_{1}\right| \geq C_{n-1} / 2 \quad \text { on } \quad\left\{\left|g_{n}\right|<\left(\delta_{n}+\eta_{n}\right) / 2\right\},
$$

and Theorem B gives us $F \in H^{\infty}$ such that

$$
\left|F-F_{1}\right|<\varepsilon \quad \text { on } \quad\left\{\left|g_{n}\right|<\eta_{n}\right\}
$$

and $C_{n} \leq|F| \leq C_{n}^{-1}$, where $C_{n}>0$ depends on $C_{n-1}, \delta_{n}, \eta_{n}$ and the constant of (4.17), which makes it clear that $C_{n}$ only has the dependencies stated in the lemma. Also, (4.18), (4.16) and (4.15) yield

$$
|F-f| \leq\left|F-F_{1}\right|+\left|F_{1}-F_{0}\right|+\left|F_{0}-f\right|<3 \varepsilon
$$

on $\bigcap_{j=1}^{n}\left\{\left|g_{j}\right|<\eta_{j}\right\}$.

Proof of Theorem A. Let $E \subset M\left(H^{\infty}\right)$ be a closed $H^{\infty}$-convex set and $f \in\left(H_{E}^{\infty}\right)^{-1}$ be an invertible function. We must show that $\hat{f}$ can be uniformly approximated on $E$ by restrictions of invertible functions in $H^{\infty}$. By the density of $\left.\hat{H}^{\infty}\right|_{E}$ in $H_{E}^{\infty}$ we can assume that $f \in H^{\infty}$ and $\inf _{E}|\hat{f}|=\gamma>0$. Consider the set

$$
K=\left\{x \in M\left(H^{\infty}\right):|\hat{f}(x)| \leq \gamma / 2\right\} .
$$

Since $E \subset M\left(H^{\infty}\right) \backslash K$ is $H^{\infty}$-convex, then for every $x \in K$ there is $g_{x} \in H^{\infty}$ with $\left\|g_{x}\right\| \leq 1$ such that $\hat{g}_{x}(x)>\sup _{E}\left|\hat{g}_{x}\right|$. Choose numbers $\delta_{x}$ and $\eta_{x}$ such that

$$
\left|\hat{g}_{x}(x)\right|>\delta_{x}>\eta_{x}>\sup _{E}\left|\hat{g}_{x}\right|
$$

The sets $V_{x}=\left\{y \in M\left(H^{\infty}\right):\left|\hat{g}_{x}(y)\right|>\delta_{x}\right\}$, with $x \in K$, form an open covering of $K$. Hence, by compactness there are $x_{1}, \ldots, x_{n} \in K$ such that $K \subset V_{x_{1}} \cup \ldots \cup V_{x_{n}}$, that is, $\{|\hat{f}| \leq \gamma / 2\} \subset \bigcup_{j=1}^{n}\left\{\left|\hat{g}_{x_{j}}\right|>\delta_{x_{j}}\right\}$. So, taking 
complements in $M\left(H^{\infty}\right)$ we have $|\hat{f}|>\gamma / 2$ on $\bigcap_{j=1}^{n}\left\{\left|\hat{g}_{x_{j}}\right| \leq \delta_{x_{j}}\right\}$. Applying Lemma 4.4 we see that given $\varepsilon>0$ there is $F \in\left(H^{\infty}\right)^{-1}$ such that

$$
|F-f|<\varepsilon \quad \text { on } \quad \bigcap_{j=1}^{n}\left\{\left|g_{x_{j}}\right|<\eta_{x_{j}}\right\} .
$$

If $G$ denotes the $M\left(H^{\infty}\right)$-closure of $\bigcap_{j=1}^{n}\left\{\left|g_{x_{j}}\right|<\eta_{x_{j}}\right\}$, by continuity, $\mid \hat{F}-$ $\hat{f} \mid \leq \varepsilon$ on $G$. The density of $\mathrm{D}$ in $M\left(H^{\infty}\right)$ and (4.19) imply that $G$ is a neighborhood of $E$. Hence, $|\hat{F}-\hat{f}| \leq \varepsilon$ on $E$ and the theorem is proved.

ACKNOWLEDGEMENTS. Both authors were partially supported by the grants MTM2005-00544 and 2005SGR00774, and the second author by the Ramón y Cajal program, from the State Secretary of Education and Universities, Spain.

\section{REFERENCES}

1. Bishop, C. J., Some characterizations of C (M), Proc. Amer. Math. Soc. 124 (1996), 26952701.

2. Carleson, L., An interpolation problem for bounded analytic functions, Amer. J. Math. 80 (1958), 921-930.

3. Carleson, L., Interpolations by bounded analytic functions and the corona theorem, Ann. of Math. 76 (1962), 547-559.

4. Corach, G., Larotonda, A. R., Stable range in Banach algebras, J. Pure Appl. Algebra 32 (1984), 289-300.

5. Corach G., and Suárez, D., Dense morphisms in commutative Banach algebras, Trans. Amer. Math. Soc. 304 (1987), 537-547.

6. Douglas, R. G., and Rudin, W., Approximation by inner functions, Pacific J. Math. 31 (1969), 313-320.

7. Folland, G. B., Introduction to Partial Differential Equations, Princeton University Press, Princeton (1976).

8. Frostman, O., Potential d'equilibre et capacité des ensembles avec quelques applications à la théorie des functions, Medd. Lunds. Univ. Mat. Sem. 3 (1935), 1-118.

9. Gamelin, T. W., Uniform Algebras, Prentice Hall, Englewood Cliffs, New Jersey (1969).

10. Garnett, J. B., Bounded Analytic Functions, Academic Press, New York (1981).

11. Garnett, J. B., and Nicolau, A., Interpolating Blaschke products generate $H^{\infty}$, Pacific J. Math. 173 (2) (1996), 501-510.

12. Jones, P., Ratios of interpolating Blaschke products, Pacific J. Math. 95 (1981), 311-321.

13. Nikolskii, N. K., Treatise on the Shift Operator, Springer-Verlag, Berlin and New York (1986).

14. Suárez, D., Čech cohomology and covering dimension for the $H^{\infty}$ maximal ideal space, J. Funct. Anal. 123 (1994), 233-263.

15. Suárez, D., Approximation by ratios of bounded analytic functions, J. Funct. Anal. 160 (1998), 254-269. 
16. Treil, S., The stable rank of $H^{\infty}$ equals 1, J. Funct. Anal. 109 (1992), 130-154.

\author{
DEPARTAMENT DE MATEMÀTIQUES \\ UNIVERSITAT AUTÒNOMA DE BARCELONA \\ 08193, BELLATERRA, BARCELONA \\ SPAIN \\ E-mail: artur@mat.uab.es,dsuarez@mat.uab.es
}

